\title{
Neutrophils are essential for induction of vaccine-like effects by antiviral monoclonal antibody immunotherapies
}

\author{
Mar Naranjo-Gomez, Jennifer Lambour, Marc Piechaczyk, and Mireia Pelegrin \\ “Equipe Labellisée par la Ligue contre le Cancer," Institut de Génétique Moléculaire de Montpellier, University of \\ Montpellier, CNRS, Montpellier, France.
}

Using a mouse retroviral model, we have shown that mAb-based immunotherapy can induce life-long endogenous protective immunity (vaccine-like effects). This observation has potentially important consequences for treating life-threatening human viral infections. Here, we investigated the role of neutrophils in this effect. Neutrophils are innate immunity effector cells with wellestablished microbe-killing activities that are rapidly mobilized upon infection. They are also emerging as orchestrators of innate and adaptive immunities. However, their immunomodulatory activity during antiviral mAb immunotherapies has never been studied. Our data reveal that neutrophils have an essential role in immunotherapy-induced immune protection of infected mice. Unexpectedly, neutrophils have a limited effect in controlling viral propagation upon passive immunotherapy administration, which is mostly mediated by NK cells. Instead, neutrophils operate as essential inducers of a potent host humoral antiviral response. Thus, neutrophils play an unexpected key role in protective immunity induction by antiviral mAbs. Our work opens approaches to improve antiviral immunotherapies, as it suggests that preserving neutrophil functions and counts might be required for achieving mAb-induced protective immunity.

Authorship note: M. Piechaczyk and M. Pelegrin are co-senior authors.

Conflict of interest: The authors have declared that no conflict of interest exists.

Submitted: September 6, 2017

Accepted: April 5, 2018

Published: May 3, 2018

Reference information:

JCI Insight. 2018;3(9):e97339. https:// doi.org/10.1172/jii.insight.97339.

\section{Introduction}

Neutralizing mAbs are now considered as a potential therapeutic approach for the prevention and treatment of chronic and acute viral infections, including newly emerging viral infections (1). In recent years, there has been a dramatic increase in the development of new mAbs with improved neutralizing activity (1-14). Notably, in addition to antirespiratory syncytial virus (RSV) mAb used to treat infant respiratory disease, several mAbs directed against human cytomegalovirus, HIV, influenza, Ebola, and rabies virus are currently engaged in promising clinical trials $(1,3,9,14)$. While the direct effects of antiviral mAbs on viral propagation have been studied extensively, little attention has been paid to their potential immunomodulatory effects until recently $(10,15,16)$.

Using a mouse model of persistent retroviral infection, i.e., the erythroleukemia induced by the murine leukemia virus, FrCasE, we have shown that treatment of infected mice with a highly neutralizing $\mathrm{mAb}$ can induce life-long protective immunity (vaccine-like effects) (see ref. 10). This observation may have major therapeutic implications for humans. Indeed, increased antiviral immune responses after mAb treatments were recently observed in preclinical models of HIV, RSV, and henipavirus infections (see ref. 10) Moreover, passive immunotherapy with broadly neutralizing mAbs stimulated antiviral antibody responses in HIV-1-infected patients (14). Similarly, vaccine-like effects have also been documented in preclinical models of cancer immunotherapies (17-19) as well as in clinical trials using anti-CD20, anti-EGFR, or anti-HER2 mAbs (20-23). Yet, neither the mechanisms at play nor the possible long-term protective consequences have been addressed in such clinical trials.

In humans, technical, ethical, and cost limitations strongly limit investigations. Instead, immunocompetent animals offer the possibility of identifying the cellular and molecular actors involved in the induction of vaccine-like effects by antiviral mAbs. The FrCasE-induced erythroleukemia is a model system permitting extensive analysis of the endogenous immune response after passive mAb-based immunotherapy under conditions of both chronic infection and pathological development. We found that a 5-day treatment of FrCasE-infected mice with the neutralizing mAb 667 (an IgG2a recognizing the retroviral envelope 
glycoprotein; Env) shortly after infection provides long-lasting (>1-year) protective antiviral immunity of the Th1 type with enhanced IgG2a humoral and cytotoxic T cell (CTL) responses and reduced regulatory $\mathrm{T}$ cell activity (24-29). The immunomodulatory action of mAb 667 depends on its ability to interact with receptors for IgGs (Fc $\gamma \mathrm{R})$. Notably, immune complexes (ICs) formed between the administered $\mathrm{mAb}$ and viral determinants enhance antiviral CTL responses through Fc $\gamma$ R-mediated binding to DCs (27).

Importantly, IC-F $\gamma$ R interactions concern several Fc $\gamma$ R-bearing innate effector cells other than DCs, including neutrophils that are rapidly recruited at sites of infections. While they have long been viewed as simple and direct scavengers of extracellular pathogens, there is recent evidence that neutrophils can be key cells in the orchestration of innate and adaptive immunity via the interaction with different myeloid and lymphoid cells and the modulation of their functions $(30,31)$. However, their role in antiviral immunity is still largely unknown. Indeed, most investigations have studied neutrophil functions in viral pathogenesis or in the control of viral propagation through their effector functions, i.e., phagocytosis, reactive oxygen species production, neutrophil extracellular traps formation, etc. $(32,33)$. In contrast, the immunomodulatory properties of neutrophils have been poorly studied in viral infections and not addressed in the context of antiviral immunotherapies.

Here, we used the FrCasE model to address the general question of neutrophil participation in the induction of protective immunity by antiviral $\mathrm{mAbs}$. We found that they are essential for the protection of infected, $667 \mathrm{mAb}$-treated mice. Unexpectedly, and unlike NK cells, neutrophils had a limited effect on viral propagation control. Instead, their protective effect relied on their capacity to induce potent humoral responses due to the acquisition of $\mathrm{B}$ cell helper activity upon $\mathrm{mAb}$ treatment. Our findings should facilitate improved design of $\mathrm{mAb}$-based antiviral therapies, as they suggest that preserving neutrophil functions and counts might be required for achieving optimal protection by $\mathrm{mAb}$.

\section{Results}

Neutrophils are necessary for protection of mice infected by antiviral mAbs without any effect on viral propagation. We first addressed neutrophil mobilization in FrCasE-infected mice with, or without, $667 \mathrm{mAb}$ treatment (infected/treated vs. infected/nontreated). Passive immunotherapy was administered on the same day after establishment of viral infection (27) (Figure 1A) and at days 2 and 5 postinfection (p.i.). Age-matched naive mice were used as controls. Neutrophil recruitment was assessed in the spleen, one of the most viremic organs, at day 8 p.i, when viral replication is maximal in infected/nontreated mice (27). Neutrophil abundance in infected/treated mice was comparable to that observed in naive mice, whereas it was significantly higher in infected/nontreated animals (Figure 1B). Interestingly, this increased neutrophil frequency was associated with a higher percentage of infected spleen cells (Figure 1C), as assessed by flow cytometry using the H34 antibody (recognizing a Gag protein epitope expressed on the surface of FrCasE-infected cells) (34-36).

Next, we assessed the role of neutrophils in the control of viral propagation as well as in the protection against leukemia in infected mice with or without $667 \mathrm{mAb}$ treatment. To this end, neutrophils were depleted by administering a mAb (1A8) directed to their specific Ly6G cell surface marker $(37,38)$ or an isotype control mAb (2A3). Depletion started 1 day before infection (Figure 1A), was efficient and specific (Supplemental Figure 1; supplemental material available online with this article; https://doi.org/10.1172/jci. insight.97339DS1), and was maintained for 21 days, i.e., the time necessary to eliminate the therapeutic 667 $\mathrm{mAb}$ (27). Neutrophil elimination accelerated disease development in infected/nontreated mice and drastically reduced protection provided by $667 \mathrm{mAb}$ to infected mice (Figure 1D). We next assessed viral propagation in the different groups of mice. In infected/nontreated animals, neutrophil depletion (Figure 2A) was associated with a significant increase in the percentage of infected spleen cells at days 8 (Figure 1E) and 14 p.i. (Supplemental Figure 2A) as well as with a higher viremia (Supplemental Figure 2B). In contrast, in infected/ treated mice, viral propagation was not significantly affected at days 8 (Figure 1E) and 14 p.i. (Figure 2B and Supplemental Figure 2) upon neutrophil depletion and remained lower than in infected/nontreated mice. This suggested that viral control by $667 \mathrm{mAb}$ involved other innate immunity effector cells.

Thus, neutrophils exert different antiviral effects on FrCasE-infected mice depending on immunotherapy. In animals undergoing simple infection, neutrophils participate in the control of viral propagation. Instead, in infected/treated mice, they are crucial during the immunotherapy period for generation of longterm protection against leukemia, despite their limited effect on viral propagation.

NK cells control viral propagation in infected/treated mice. As NK cells can exert antibody-dependent cellular cytotoxicity (ADCC) activity against infected cells exposing determinants such as the retroviral Env protein 
A
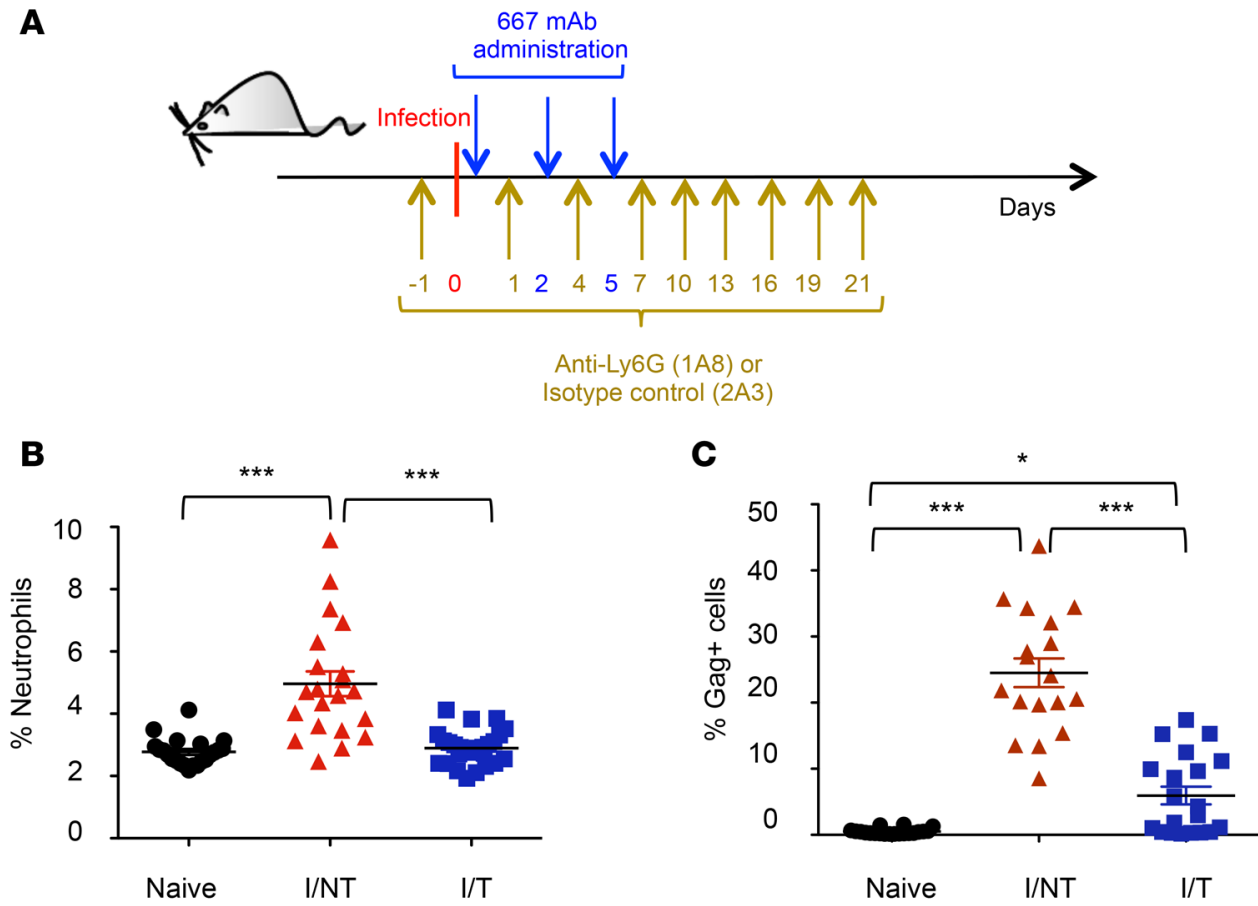

D

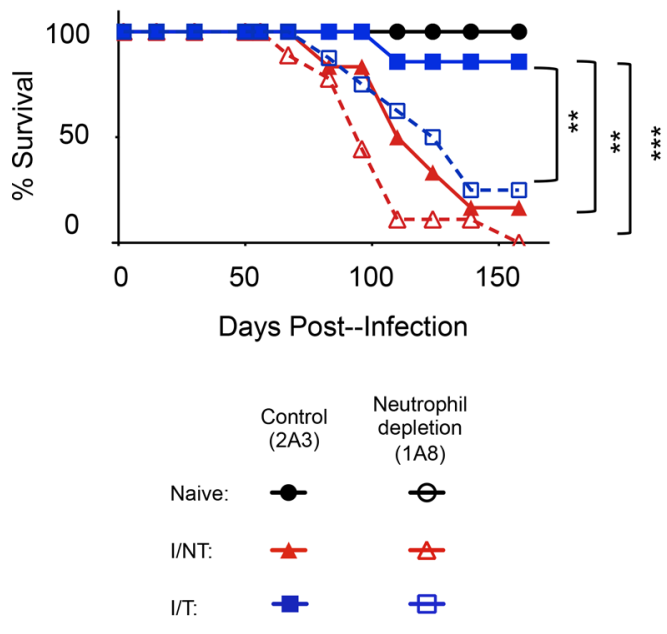

$\mathbf{E}$

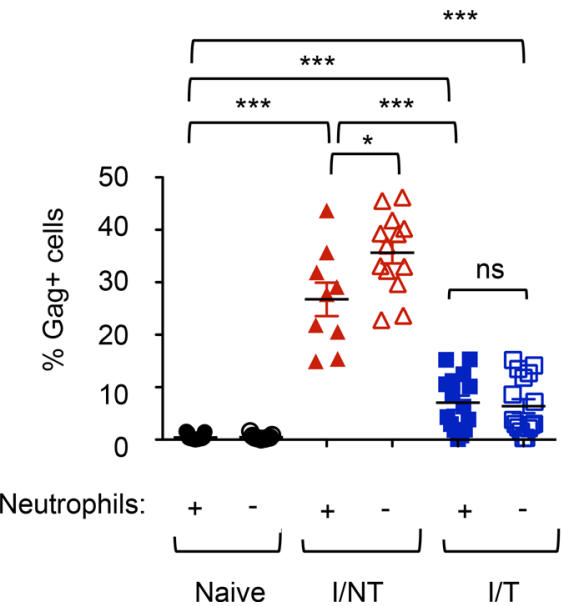

Figure 1. Antiviral effects of neutrophils. (A) Experimental scheme. Eight-day-old pups were infected and treated with the $667 \mathrm{mAb}$ as indicated. Mice were treated as indicated with the anti-Ly6C $1 A 8$ mAb or the isotype control $2 A 3 \mathrm{mAb}$ in neutrophil depletion experiments. (B and $\mathbf{C}$ ) Neutrophil recruitment and infected cell rate in spleen. Splenocytes from naive, infected/nontreated (I/NT), and infected/ treated $(\mathrm{I} / \mathrm{T})$ mice were analyzed by flow cytometry on day 8 p.i. for (a) neutrophil recruitment (percentage of $\mathrm{Ly}_{\mathrm{G}} \mathrm{G}^{+}$cells) and (b) retroviral positivity of splenocytes (percentage of $\mathrm{Gag}^{+}$ cells) gated in the CD45.2+ population. The data presented correspond to 5 independent experiments, with at least 15 mice per group. (D) Mouse survival. Naive, I/NT, and I/T mice were treated with either the antineutrophil (1A8) or the control (2A3) $\mathrm{mAb}$ as indicated in $A$ and followed up for leukemic death. The data represent 2 independent experiments, with 6-9 mice per group. (E) Infected cells rate upon neutrophil depletion. Neutrophils of naive, I/NT, and I/T mice were depleted, or not, as indicated in $\mathbf{A}$ and infected splenocytes were assayed as in $\mathbf{C}$ on day 8 p.i. The data represent 4 independent experiments, with 9-15 mice per group. Data are expressed as mean \pm SEM. Statistical significance was established using a parametric 1-way ANOVA test with a Bonferroni correction $\left({ }^{*} P<0.05\right.$; ${ }^{* *} P<0.01$; ${ }^{* *} P<0.001$ ).

$(27,39,40)$, we asked whether NK cells were involved in the control of viral propagation in infected/treated mice. To this end, NK cells were depleted using an anti-asialo-GM1 antibody (41-44) (Figure 2A). In contrast to neutrophil depletion, the absence of NK cells in infected/treated mice led to a significantly increased viral propagation at day 14 p.i. (Figure 2B). Next, we compared $667 \mathrm{mAb}$-mediated ADCC activity of NK cells and neutrophils with infected cells using an in vivo antibody-mediated killing assay, relying on the administration of $667 \mathrm{mAb}$-opsonized FrCasE-infected splenocytes in naive mice (27) (Figure 2C). Depletion of NK cells, but not neutrophils, led to reduced mAb-mediated infected cell lysis (Figure 2D). Finally, as NK cells are crucial for viral propagation control during the immunotherapy period, we assessed their role in the protection against leukemia in infected/treated mice. Importantly, NK cell depletion drastically reduced survival of immunotreated mice (Figure 2E), indicating that antibody-mediated control of viral propagation by NK cells is necessary for long-term antiviral protection.

Thus, NK cells are crucial for protection of infected/treated mice via efficient control of viral propagation by $667 \mathrm{mAb}$-mediated ADCC.

Neutrophils differentially alter innate lymphoid cell biology in infected/treated and infected/nontreated mice. Innate lymphoid cells (ILCs) are a heterogeneous population of immune cells that includes NK cells and 
A

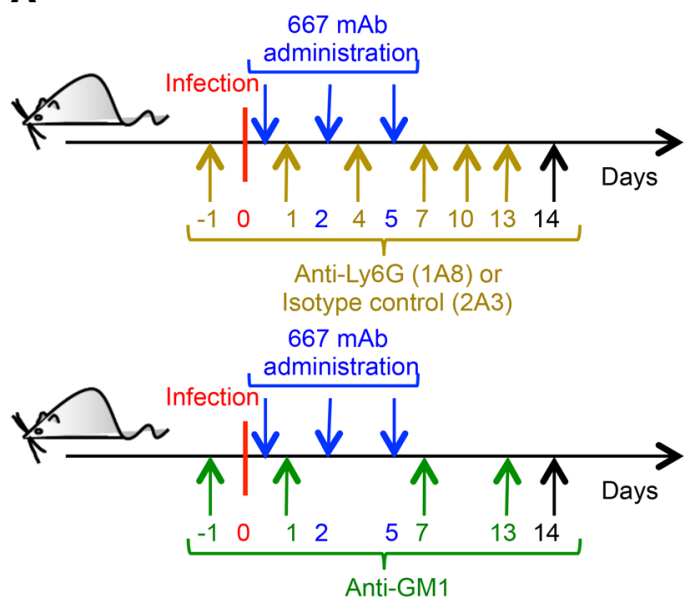

C

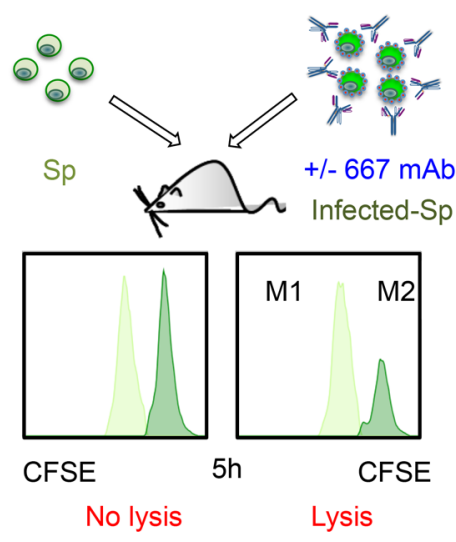

B
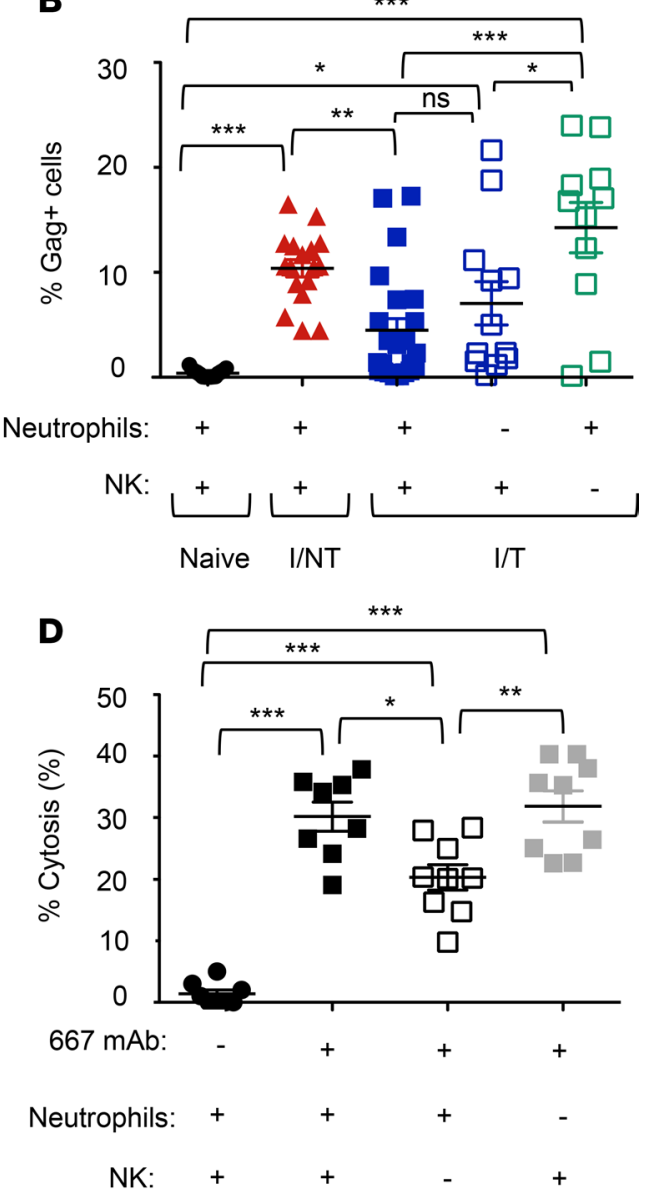

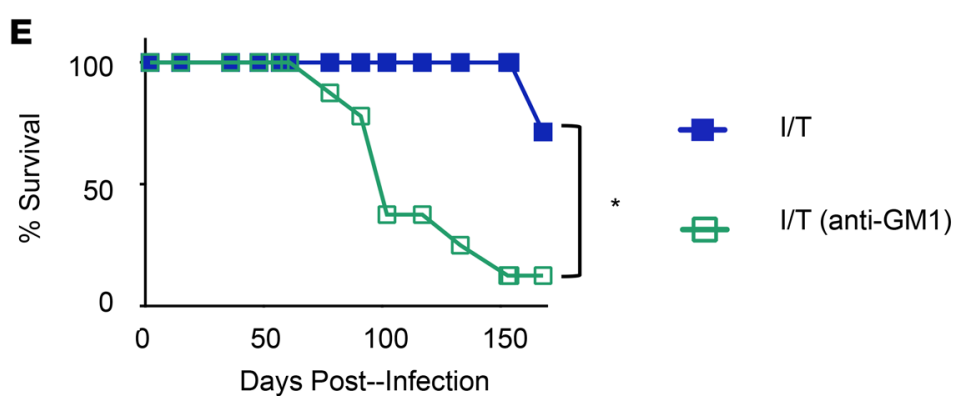

Figure 2. Antibody-mediated control of viral propagation by NK cells. (A) Experimental scheme. Mice were infected and $m A b$-treated as in Figure 1A. Infected/treated mice were treated as indicated with the anti-Ly6C 1A8 mAb or the isotype control 2A3 mAb to deplete neutrophils and infected/treated mice were treated as indicated with the anti-asialo-GM1 antibody to deplete NK cells. (B) Effect of neutrophils or NK cell depletion in viral spread in infected/treated mice. Percentage of infected cells at day $14 \mathrm{p.i}$. in the spleens of naive, I/NT, and I/T mice, depleted or not of neutrophils or NK cells assessed as in Figure 1C. The data represent 3 independent experiments, with at least 8 mice per group. (C and D) In vivo cytolysis activity of $667 \mathrm{mAb}$ in naive mice after depletion of neutrophils or NK cells. Splenocytes from noninfected mice (Sp) were labeled using $0.5 \mu \mathrm{M}$ of the vital dye CFSE (CFSE ${ }^{10}$ cells; M1) and mixed at a 1:1 ratio with splenocytes from infected mice (Infected-Sp) labeled using $5 \mu \mathrm{M}$ CFSE (CFSE hi cells; M2) and preincubated, or not, with $667 \mathrm{mAb}$. Mixed cell populations were administered to naive mice 1 day after depletion of either neutrophils or NK cells with the $1 \mathrm{~A} 8 \mathrm{mAb}$ or the anti-asialo-GM1 antibody, respectively. Cytolysis was quantified 5 hours later, as described in Methods section. The data are presented as mean \pm SEM of 2 independent experiments, with at least 8 mice per group. Statistical significance was established using a parametric 1-way ANOVA test with a Bonferroni correction (B and D). (E) Effect of NK cell depletion in the survival of infected/ treated mice. I/T, NK cells, depleted or not as indicated in $\mathbf{A}$, were followed up for leukemic death. The data represent 2 independent experiments, with 7 mice per group. Statistical significance was established using an unpaired Student's $t$ test. Data are expressed as mean \pm SEM $\left({ }^{*} P<0.05\right.$, $\left.{ }^{* *} P<0.01,{ }^{* * *} P<0.001\right)$. 
A
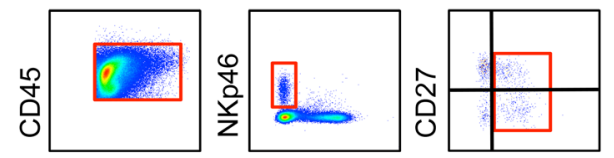

CD11b

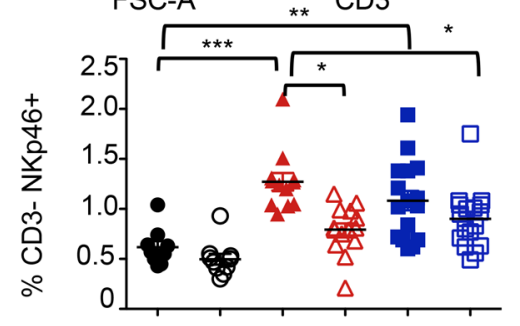

Neutrophils:
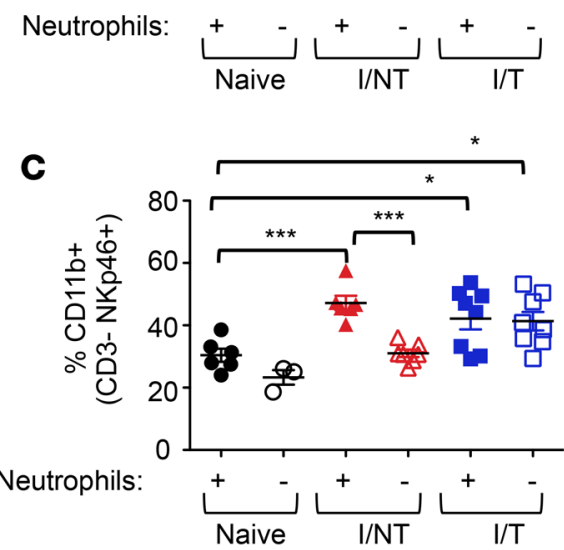

D
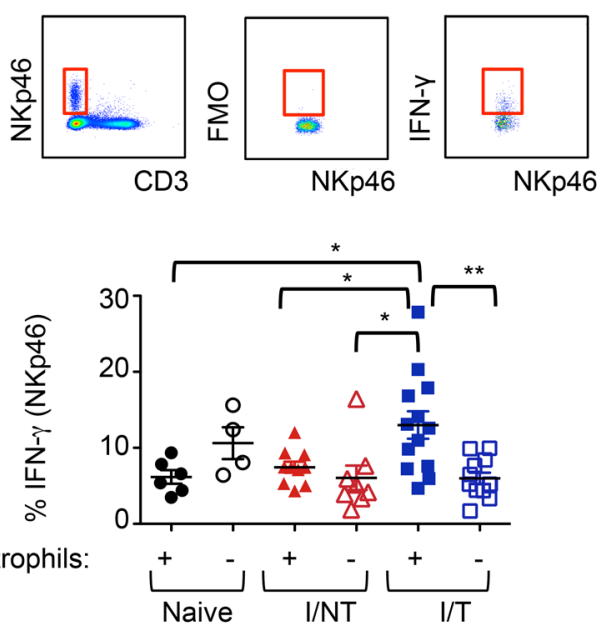

B
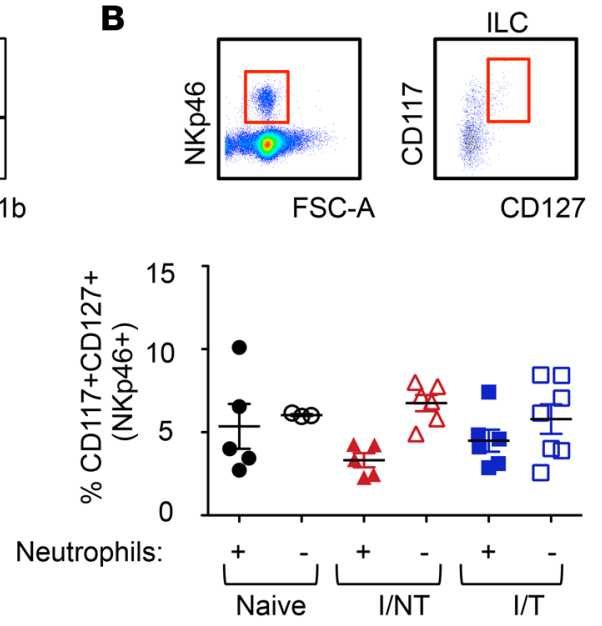

E
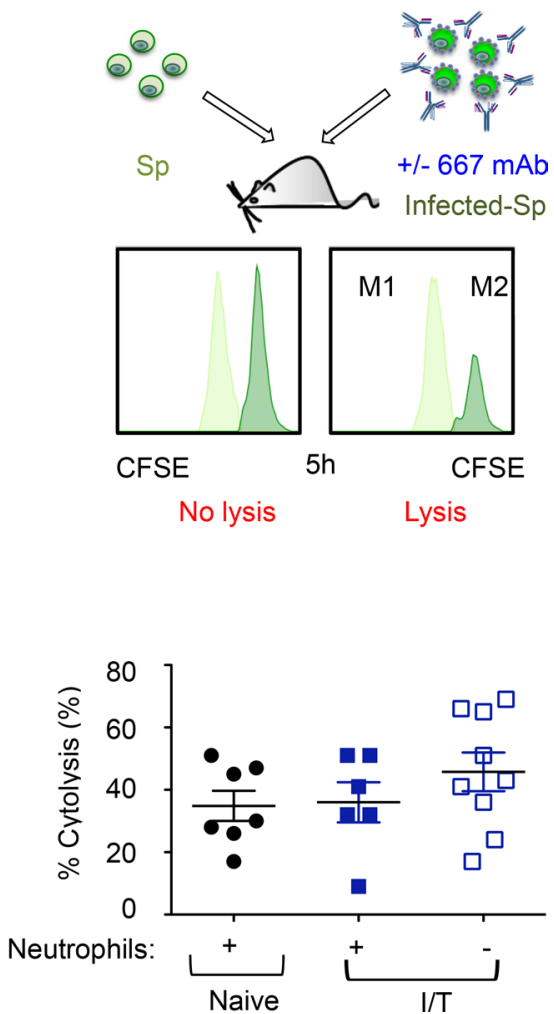

Figure 3. Effects of neutrophil depletion on innate lymphoid cell recruitment and biology. (A-D) Neutrophils of naive, I/ NT, and I/T mice were depleted, or not, as indicated in Figure 1A, and ILC in the spleen were assayed 14 days p.i. by flow cytometry. (A) Frequency of CD3-NKp46 ${ }^{+}$ cells in the CD45.2+ leukocytic population (B) Frequency of $\mathrm{CD} 117^{+} / \mathrm{CD} 127^{+}$cells in the Lin-NKp46+ population. (C) Maturation (CD11b+ cells) and (D) expression of IFN- $\gamma$ in the $\mathrm{CD}^{-}{ }^{-N K p} 46^{+}$population. (E) In vivo cytolysis activity of $667 \mathrm{mAb}$ in infected/treated mice after depletion of neutrophils. The $1 A 8$, or the $2 A 3$ isotype control $\mathrm{mAb}$, was administered to I/T mice, and 667 ADCC activity was quantified at 30 days p.i., as in Figure 2, C and $D$. The data represent at least 2 independent experiments. Data are expressed as mean \pm SEM. Statistical significance was established using a parametric 1-way ANOVA test with a Bonferroni correction ( $\left.{ }^{*} P<0.05 ;{ }^{* *} P<0.01 ;{ }^{* * *} P<0.001\right)$.

ILC1, ILC2, and ILC3. As NK biology can be affected by neutrophils (45), we first assessed the effect of neutrophil depletion on NK cells. To this end, we measured the frequency of CD3-NKp46 ${ }^{+}$cells in the spleens of mice from the different groups. The $\mathrm{CD}^{-} \mathrm{Nkp}^{-} 6^{+}$population contains mostly NK cells but may also include ILC1 and a subpopulation of ILC3 cells. NK cells can be distinguished from such ILCs using the cell surface marker CD49b (46). Notably, over $95 \%$ of CD3-NKp46 ${ }^{+}$cells were CD49b ${ }^{+}$(Supplemental Figure 3), indicating that the vast majority of $\mathrm{CD}^{-} \mathrm{NKp} 46^{+}$cells in the spleen displayed a NK phenotype. As shown in Figure 3A, CD3-NKp46 $6^{+}$cell recruitment in spleens of both infected/nontreated and infected/ treated mice at day 14 p.i. was similar and stronger than in naive mice. We also observed that neutrophils were involved in $\mathrm{CD} 3-\mathrm{NKp} 46^{+}$cell mobilization in infected/nontreated mice but not in infected/treated or in naive mice (Figure 3A). We next assessed the frequency of splenic ILCs other than NK cells by quantifying CD117 and CD127 expression in the $\mathrm{NKp} 46^{+}$spleen cell population lacking the common lymphoid and myeloid lineage-associated (Lin-associated) markers ( $\left.\operatorname{Lin}^{-}\right)(47)$. The recruitment of those ILCs in spleens 
of both infected/nontreated and infected/treated mice at day 14 p.i. was not significantly different from that observed in naive mice (Figure 3B). In addition, neutrophil depletion did not significantly alter the frequency of such ILCs (Figure 3B) in any group of mice.

These results show an enhanced recruitment of $\mathrm{CD}^{-} \mathrm{NKp} 46^{+}$cells, which are mostly NK cells, in both infected/treated and infected/nontreated mice, but these effects are neutrophil dependent only in the latter. They also show that neutrophil depletion does not affect the frequency of splenic in $^{-} \mathrm{CD} 117^{+} \mathrm{CD} 127^{+} \mathrm{ILCs}$, in agreement with the lack of effect of anti-Ly6G-mediated neutrophil depletion on splenic ILCs reported in other experimental settings (47).

To better characterize the effect of neutrophils on splenic CD3-NKp46 ${ }^{+}$cells, we assessed their maturation by monitoring $\mathrm{CD} 11 \mathrm{~b}$ and $\mathrm{CD} 27$ expression at day 14 p.i. These markers identify different stages of NK maturation in mice (45). Relative to naive mice, both infected/nontreated and infected/treated mice showed similar higher frequencies of $\mathrm{CD} 11 \mathrm{~b}^{+} \mathrm{CD} 3^{-} \mathrm{NKp} 46^{+}$cells (including fully mature $\mathrm{CD} 11 \mathrm{~b}^{+} \mathrm{CD} 27^{-}$ and semimature $\mathrm{CD} 11 \mathrm{~b}^{+} \mathrm{CD} 27^{+}$cells) (Figure $3 \mathrm{C}$ ) at the expense of immature ones (CD11b ) (data not shown). Moreover, depletion of neutrophils entailed a strong reduction of the frequency of $\mathrm{CD}^{-1} \mathrm{~b}^{+} \mathrm{CD}^{-}$ $\mathrm{NKp} 46^{+}$cells only in infected/nontreated mice. Further characterization of $\mathrm{CD}^{-} \mathrm{NKp} 46^{+}$cells indicated higher neutrophil-dependent IFN- $\gamma$ production in infected/treated mice but low and similar IFN- $\gamma$ levels in naive and infected/nontreated animals, whether the latter were neutrophil proficient or deficient (Figure 3D). Finally, we assessed whether neutrophils affected the ADCC activity of NK cells in infected/ treated mice. Notably, ablation of neutrophils did not alter $667 \mathrm{mAb}$-mediated ADCC activity of NK cells (Figure 3E), consistent with the lack of effect on viral propagation (Figure 1E and Figure 2B).

Thus, CD3 ${ }^{-} \mathrm{NKp} 46^{+}$maturation is similarly enhanced in both infected/treated and infected/nontreated mice, but these effects are neutrophil dependent only in the latter. Nevertheless, neutrophils might participate in functional activation of $\mathrm{CD}^{-} \mathrm{NKp}^{-} 6^{+}$cells in infected/treated animals, as our results show that neutrophils modulate their IFN- $\gamma$ secretion capacity in infected/treated mice.

Neutrophils are crucial for inducing the humoral, but not the $C D 8^{+} T$ cell, antiviral response in immunotherapy-treated mice. Since neutrophils exert no control over viral propagation during the immunotherapy period (Figure 1E and Figure 2B) but are necessary for long-term protection of mice (Figure 1D), we asked whether they could be key for the induction of vaccine-like effects.

We first addressed cellular adaptive immunity by assaying the primary virus-specific $\mathrm{CD} 8^{+} \mathrm{T}$ cell response in infected/nontreated and infected/treated mice at its peak (i.e., 14 days p.i.) (27) with or without neutrophil depletion. Consistent with our previous work, infected/treated mice showed an increased virus-specific $\mathrm{CD}^{+}$ $\mathrm{T}$ cell response relative to infected/nontreated mice (27). In neither case was the frequency of virus-specific $\mathrm{CD}^{+} \mathrm{T}$ cells altered by neutrophil depletion (Figure $4 \mathrm{~A}$ ). Similarly, neither case showed any difference in the frequency of $\mathrm{CD}^{+} \mathrm{T}$ cells expressing IFN- $\gamma$ (Figure 4B). These data ruled out a major role for neutrophils in the $667 \mathrm{mAb}$-induced antiviral $\mathrm{CD} 8^{+} \mathrm{T}$ cell response.

Next, we addressed humoral immunity in infected/nontreated and infected/treated mice depleted or not of neutrophils. To achieve this, anti-FrCasE serum Igs from mice of the different groups were assayed by ELISA. Virus-specific IgM titers were assayed at 14 days p.i. (i.e., at the peak of the IgM response upon FrCasE infection and $667 \mathrm{mAb}$ treatment; Supplemental Figure 4) and were not significantly different between infected/nontreated and infected/treated mice (Figure 5A). Interestingly, neutrophil depletion did not alter virus-specific IgM titers in either infected/nontreated or infected/treated mice (Figure $5 \mathrm{~A}$ ). In agreement with published studies in other experimental settings, the absence of neutrophils did not affect the levels of $\operatorname{IgM}(48)$. On the contrary, and consistent with our previous work (27), infected/treated mice displayed a much higher level of anti-FrCasE IgGs than infected/nontreated animals (Figure 5B), with a peak $(700 \mu \mathrm{g} / \mathrm{ml})$ by day 68 p.i. and a still high level $(100 \mu \mathrm{g} / \mathrm{ml})$ at the end of the experiment. Interestingly, in the absence of neutrophils, serum concentration of antiviral IgGs dramatically decreased in infected/ treated mice (Figure 5B and Supplemental Figure 5). In contrast, neutrophils showed no role in the regulation of the poor nonprotective antibody response elicited in infected/nontreated mice (Figure 5B). Importantly, high anti-FrCasE IgG seric levels correlated with longer survival times, supporting a role for the high humoral antiviral response in protection against disease (Figure $5 \mathrm{C}$ ). To further characterize the long-term virus-specific humoral response, we tested whether infected/treated mice, depleted or not of neutrophils, could respond to a virus challenge performed 3 months after the first infection (i.e., a time point at which the primary humoral response has strongly declined). Infected/treated mice, depleted or not of neutrophils, were inoculated with FrCasE, and serum samples were collected 1 week later to assay the generation of 
A

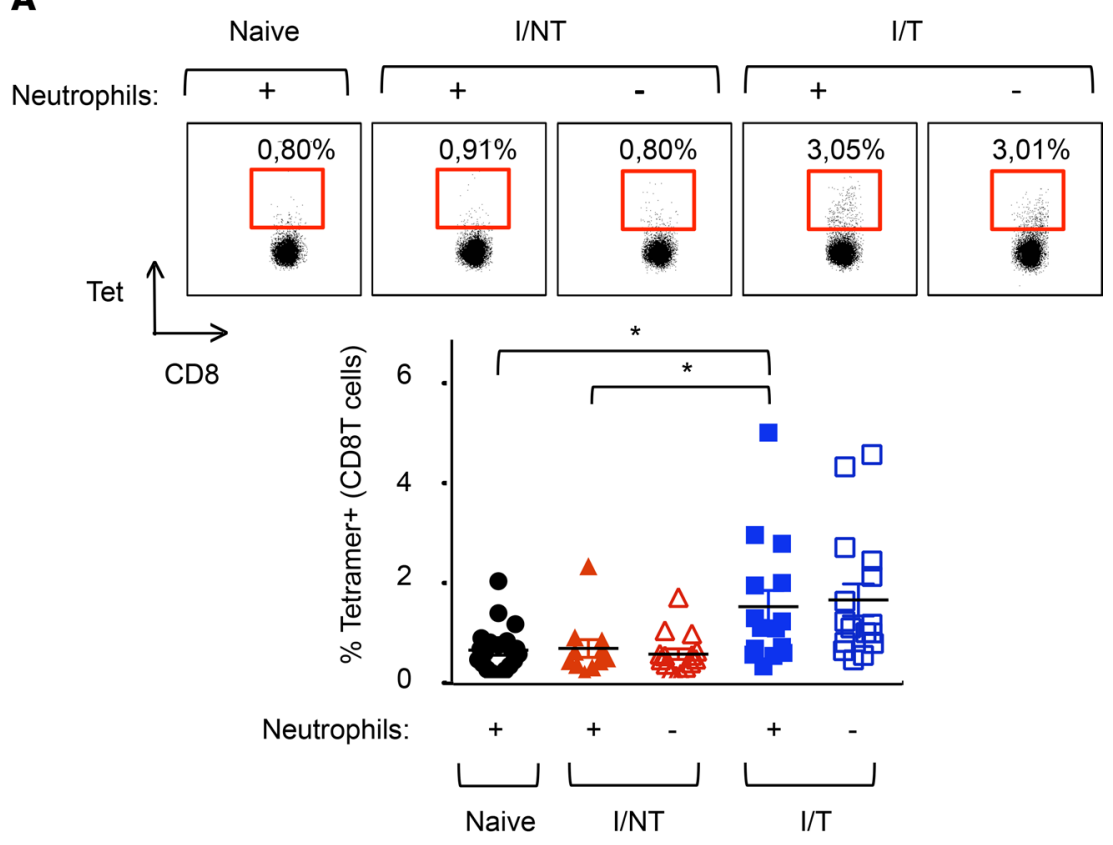

Figure 4. Assay of FrCasE-specific CD8+ $\mathrm{T}$ cells in the presence and absence of neutrophils. (A) Neutrophils of naive, I/NT, and I/T mice were depleted, or not, as indicated in Figure 1A. Frequency of FrCasE-specific CD8 ${ }^{+} T$ cells. Spleen cells were isolated at day 14 p.i., and the frequency of virus-specific CD8 ${ }^{+} T$ cells in the total $C D 8^{+} T$ cells population was assayed by flow cytometry using the $\mathrm{H}_{2} \mathrm{D}^{\mathrm{b}}$-GagL MHC tetramer. The data represent 4 independent experiments, with at least 11 mice per group. (B) Expression of IFN $-\gamma$ by $\mathrm{CD}^{+} \mathrm{T}$ cells. Splenic CD8 ${ }^{+} \mathrm{T}$ cells were analyzed by flow cytometry for the expression of IFN- $\gamma$. The data presented represent 3 independent experiments, with at least 7 mice per group. Data are expressed as mean \pm SEM. Statistical significance was established using a parametric 1-way ANOVA test with a Bonferroni correction $\left({ }^{*} P<0.05\right)$.

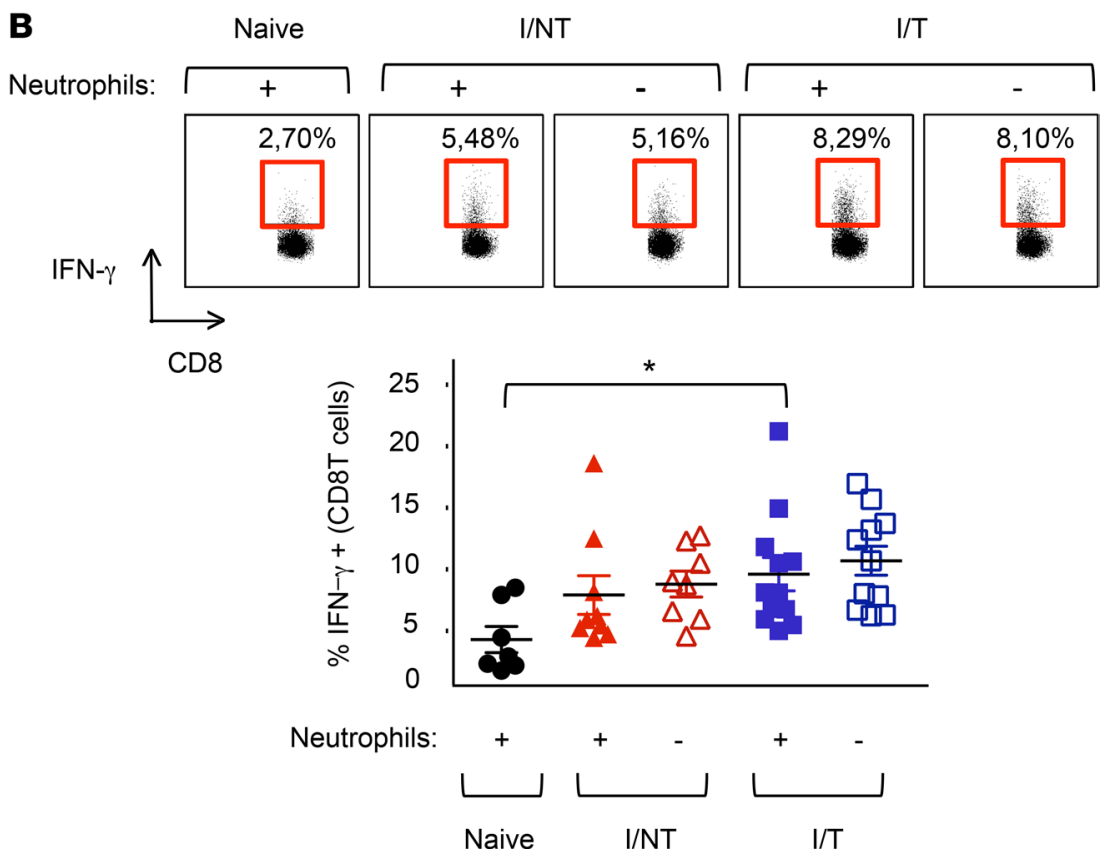

endogenous anti-FrCasE antibodies. In the presence of neutrophils, 3 of 5 infected/treated mice showed an increase in the virus-specific humoral response (Figure 5D), while, in the absence of neutrophils, none of the infected/treated mice responded to the viral challenge. Altogether, these results show that neutrophil depletion at early time points after infection and treatment affects humoral immunity with an effect on both primary and memory virus-specific B cell responses.

As marginal zone (MZ) B cells are known to contribute to the mounting of antibody responses $(49,50)$ and their function might be modulated by neutrophils $(51)$, we next addressed the frequencies of splenic MZ (CD21 ${ }^{\mathrm{hi}} \operatorname{IgM}^{\mathrm{hi}} \mathrm{CD} 19^{+}$cells) and follicular (FO) (CD23+ $\operatorname{IgM}^{\mathrm{lo}} \mathrm{CD} 19^{+}$cells) B cells. MZ B cell frequency was enhanced in a neutrophil-dependent manner in infected/treated mice but unchanged in infected/nontreated animals relative to naive mice (Figure 6A). In contrast, the frequency of FO B cells was significantly enhanced in infected/treated mice relative to that in naive mice; however, there was no role for neutrophils (Figure 6B). Interestingly, consistent with a role of MZ 

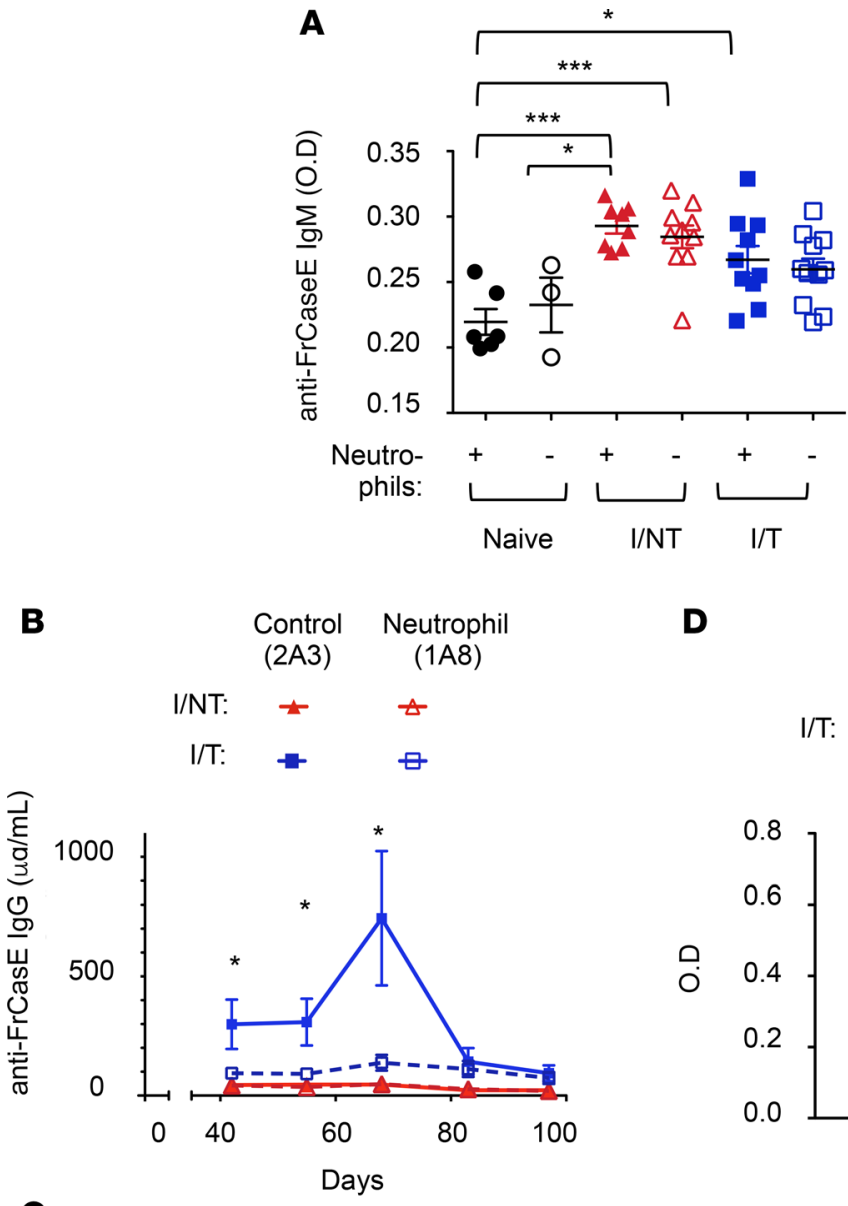

C

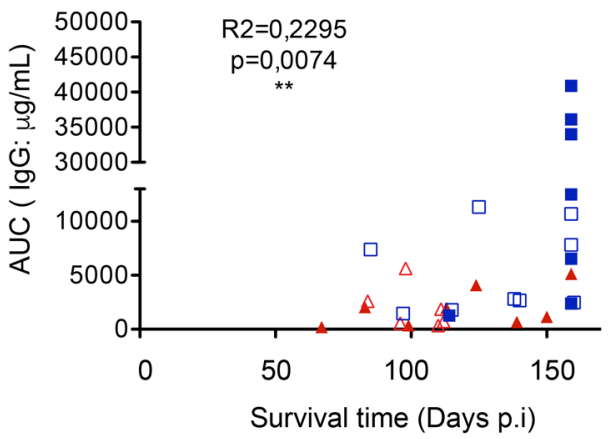

D
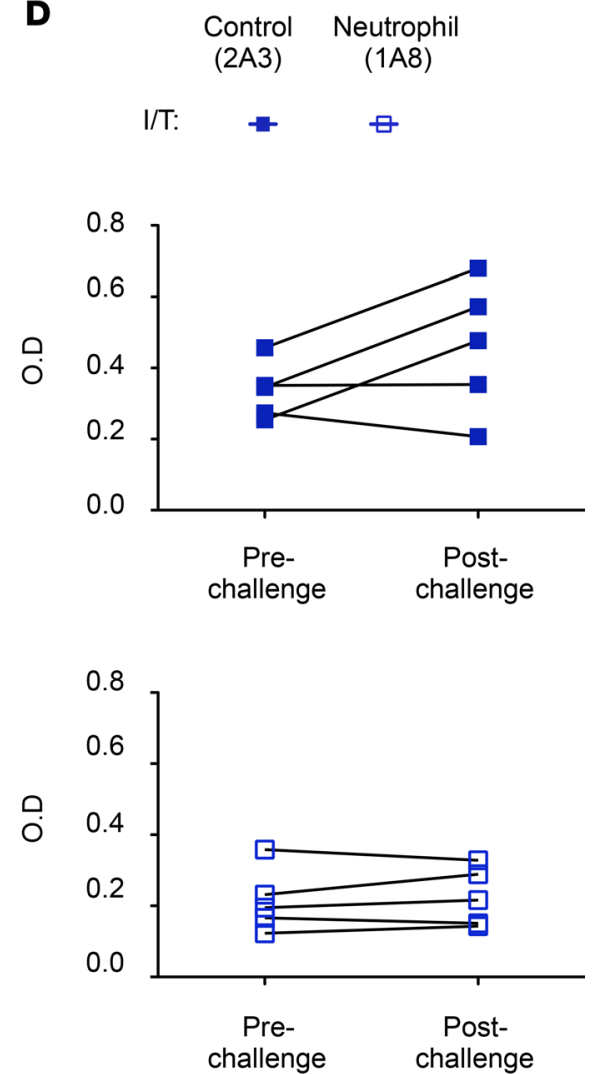

Figure 5. Enhancement of the humoral antiviral response by neutrophils. Neutrophils of naive, I/NT, and I/T mice were depleted, or not, as indicated in Figure 1A. (A and B) Serum concentration of FrCasE-specific Igs. (A) Seric FrCasE-specific IgM levels were assayed by ELISA at 14 days p.i. The data represent 2 independent experiments, with 8-11 mice per group (for I/NT and I/T mice) and 3-6 mice per group (for naive mice). (B) Seric FrCasE-specific IgC concentration was assayed by ELISA at the indicated times. The data represent 2 independent experiments, with 7-9 mice per group. Data are expressed as mean \pm SEM. Statistical significance was established using a parametric 1-way ANOVA test, with a Bonferroni correction. (C) Correlation between serum anti-FrCasE IgC levels (evaluated as AUC) and survival times, analyzed using the Pearson correlation test. AUC was evaluated until the last time point at which all mice were still alive (day 68 p.i.). All infected/nontreated $(n=$ $8)$ and infected/treated $(n=9)$ mice, depleted or not of neutrophils ( $n=9$ and $n=7$, respectively), showed in Figure 1D were evaluated for such a correlation. (D) FrCasE-specific secondary humoral response. Seric FrCasE-specific IgG levels in I/T mice (depleted or not of neutrophils) were assayed by ELISA before and 1 week after a viral challenge performed at day 93 p.i. The data represent 2 independent experiments, with 5 mice per group. Statistical significance was established using a paired Student's $t$ test $\left({ }^{*} P<0.05\right.$; ${ }^{* *} P<0.01$; $\left.{ }^{* * *} P<0.001\right)$

B cells in the generation of plasma cells (49), the higher frequency of splenic MZ B cells at day 14 p.i. in infected/treated mice was associated with a higher frequency of CD $138^{+}$plasma cells in bone marrow (BM), as compared with infected/nontreated animals. This effect was neutrophil dependent (Figure 6C). Finally, histological analyses of spleens of infected mice at 14 days p.i. revealed larger germinal centers (GCs; defined by staining of MZ CD169+ macrophages and B220 $0^{+}$cells) in infected/ treated versus infected/nontreated mice (Figure 6D). In agreement with a role of neutrophils in the enhancement of the humoral response, neutrophil depletion in infected/treated mice led to smaller GCs (Figure 6D).

Thus, neutrophils are essential for the vaccine-like effects induced by the $667 \mathrm{mAb}$ immunotherapy through the stimulation of the humoral, but not the $\mathrm{CD} 8^{+} \mathrm{T}$ cell, antiviral response. This effect is associated with a neutrophil-dependent increase in both splenic MZ B cells and BM plasma cells.

Neutrophils are differently activated in infected/treated and nontreated mice. Finally, we addressed the functional activation of splenic neutrophils in infected mice with or without $667 \mathrm{mAb}$ immunotherapy at day 
A
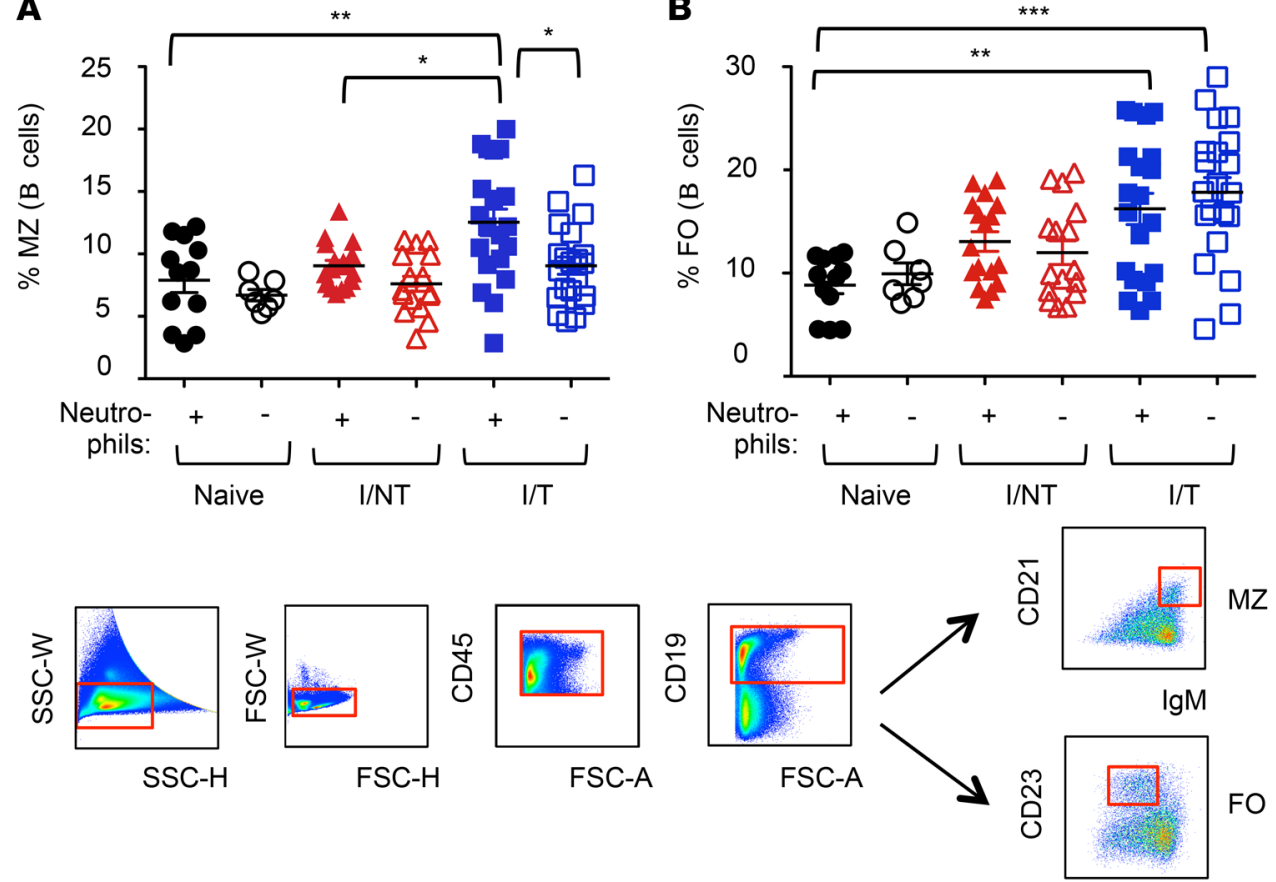

B

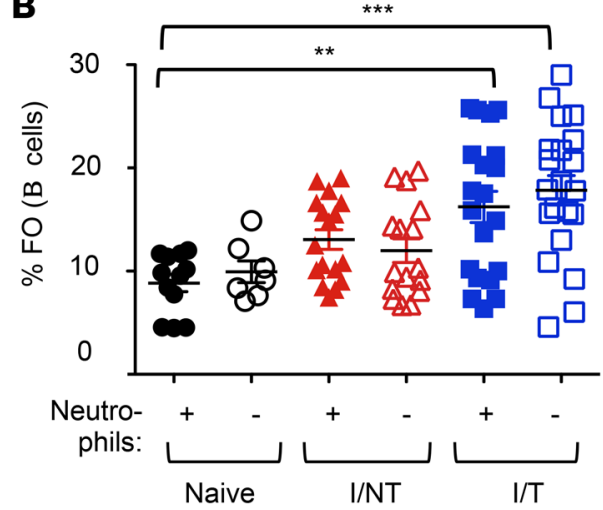

$\lg M$

C

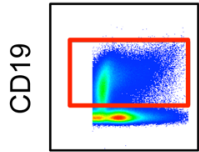

FSC-A

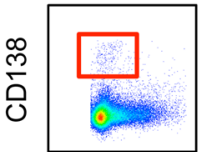

FSC-A
D
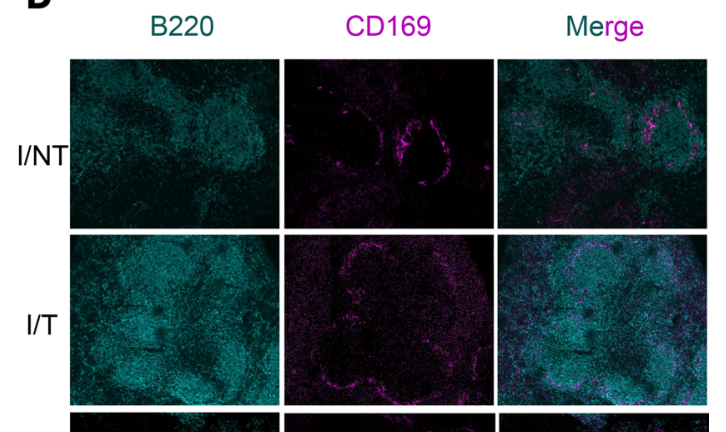
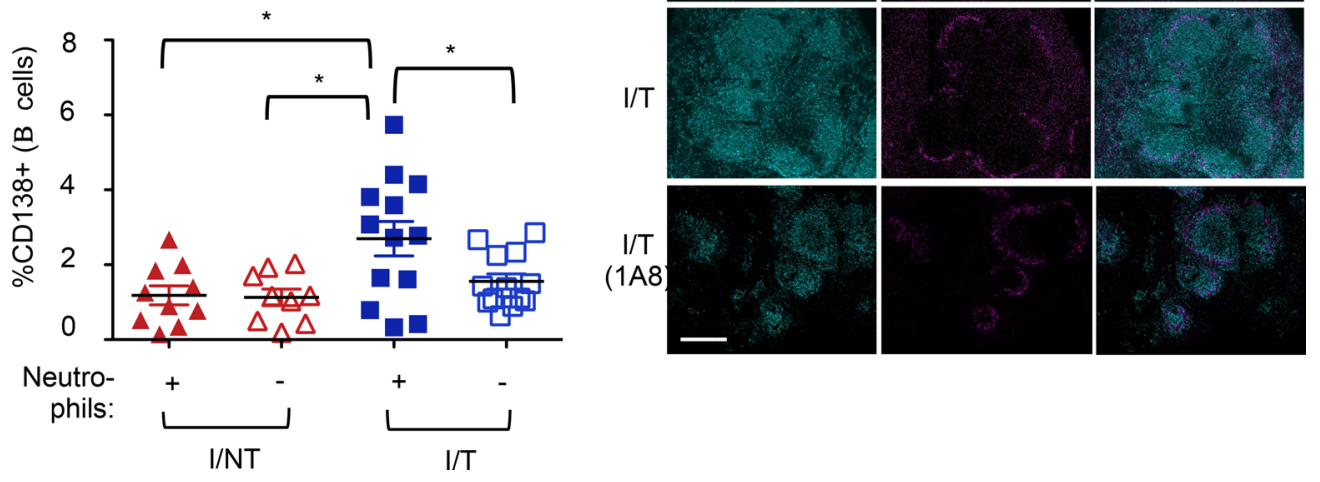

Figure 6. Effects of neutrophil depletion on B cell responses. Neutrophils of naive, I/NT, and I/T mice were depleted, or not, as indicated in Figure 1A. (A and $\mathbf{B}$ ) Frequency of $\mathrm{MZ}$ and follicular (FO) B cells. Spleen cells were isolated at day 14 p.i. and were analyzed by flow cytometry for the frequency of $M Z$ (CD21 ${ }^{\text {hil }} g M^{\text {hi }}$ ) (A) and $\mathrm{FO}\left(\mathrm{CD}^{2} 3^{+} \mathrm{IgM}^{\mathrm{l}}\right)$ (B) $\mathrm{CD}^{\circ} 9^{+} \mathrm{B}$ cells, as depicted in the gating strategy. (C) Frequency of plasma cells. BM cells were isolated at day 14 p.i. and were analyzed by flow cytometry for the frequency of $\mathrm{CD}^{3} 8^{+}$(CD19+) B cells. The data represent 5 independent experiments, with 7-12 mice per group for naive mice and 17-21 per group for I/NT and I/T mice. Data are expressed as mean \pm SEM. Statistical significance was established using a parametric 1-way ANOVA test with a Bonferroni correction ( ${ }^{*} P$ $<0.05$; ${ }^{*} P<0.01$; $\left.{ }^{* *} P<0.001\right)$.

(D) Histological analyses of spleen sections. Immunolabeling of B cells $\left(\mathrm{B} 22 \mathrm{O}^{+}\right)$and macrophages of the $\mathrm{MZ}$ (CD169') was performed in sections from spleens of infected/nontreated and infected/treated mice (depleted or not of neutrophils) recovered at 14 days p.i. to visualize germinal centers. The images are representative of 4 separate mice for each experimental condition. Scale bar: $200 \mu \mathrm{m}$.

8 p.i. by monitoring cell surface activation markers and quantifying various cytokine mRNA and protein levels. In infected/treated and infected/nontreated mice, splenic neutrophils were similarly activated, as deduced from CD62L shedding and increased expression of CD11b (Figure 7A). However, neutrophils from infected/treated mice showed a significantly stronger expression of two cytokines involved in B cell activation, B cell-activating factor (BAFF) and lymphotoxin $\alpha$ (LT $\alpha)$, compared with infected/nontreated animals (Figure 7B), suggesting a role for the therapeutic $\mathrm{mAb}$ in the functional activation of neutrophils. Splenic neutrophils sorted from infected/treated mice also showed a trend for higher secretion capacity of BAFF and LT $\alpha$, as compared with infected/nontreated mice neutrophils, even though such a trend did not reach statistical significance. To further assess whether $\mathrm{mAb}$ triggering (through Fc $\mathrm{R}$ cross-linking) could enhance BAFF and LT $\alpha$ release by neutrophils, we isolated BM neutrophils from naive mice and stimulated them for 24 hours in plates coated with the $667 \mathrm{mAb}$. Experiments were conducted in the presence or the absence of the proinflammatory cytokine IFN- $\gamma$ to assess the extent to which inflammatory conditions could synergize with $667 \mathrm{mAb}$ triggering. Interestingly, in the presence of IFN- $\gamma, 667 \mathrm{mAb}$ triggering led to a significantly increased release of BAFF and LT $\alpha$ by neutrophils (Figure 7C). 
A
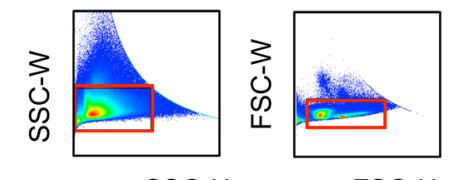

SSC-H

FSC-H
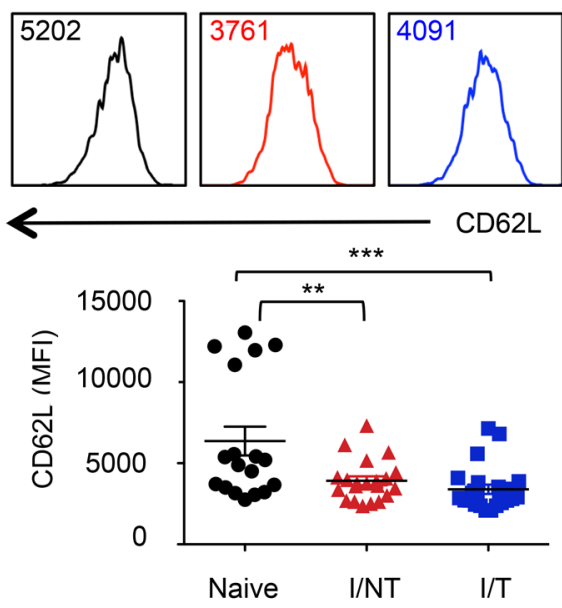
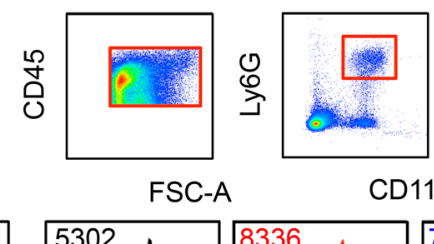

CD11b
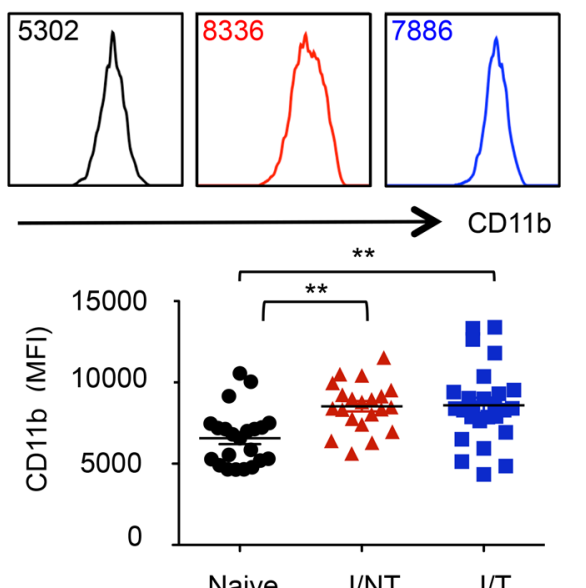

B Infected/non-treated $\bigcirc$ Infected/treated
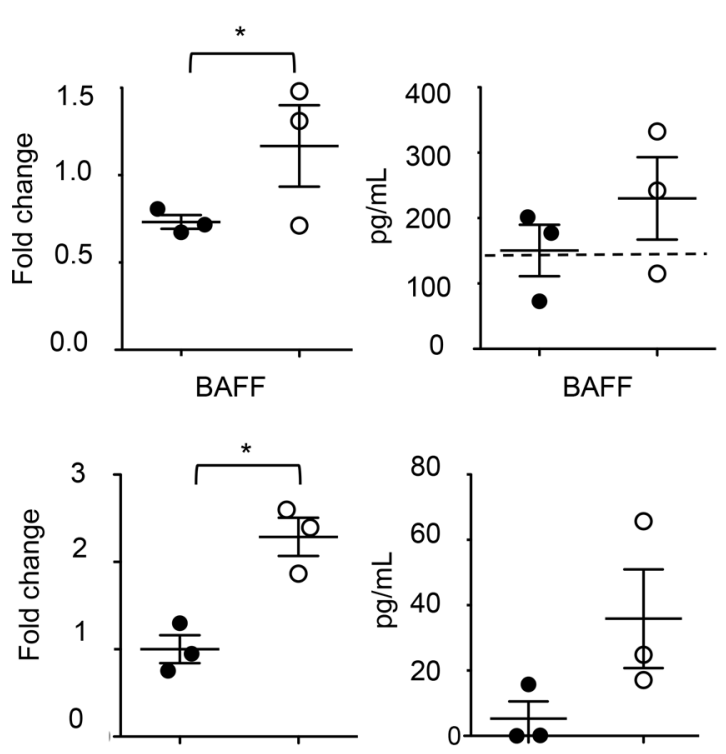

Lt $\alpha$

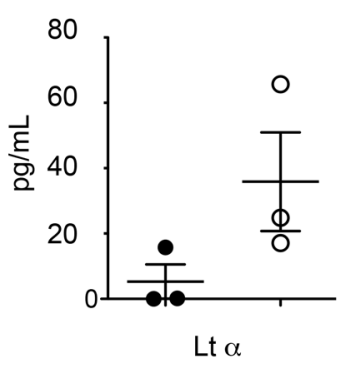

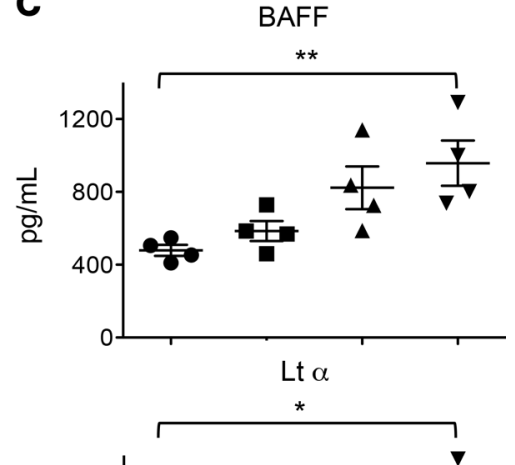

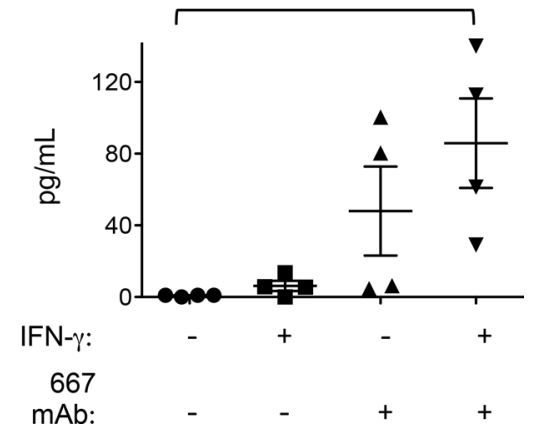

Figure 7. Activation of splenic and BM-isolated neutrophils. (A) Expression of CD11b and CD62L. Spleen cells from naive, I/ $\mathrm{NT}$, and $\mathrm{I} / \mathrm{T}$ mice were isolated at day $8 \mathrm{p.i}$. and were analyzed by flow cytometry for assaying cell surface expression of CD11b and CD62L. The data represent 5 independent experiments, with at least 18 mice per group. Data are expressed as mean \pm SEM. (B) Expression and protein release of BAFF and LT $\alpha$ by neutrophils. Neutrophils from naive, I/NT, and I/T mice were sorted from spleens at day 8 p.i. and assessed for cytokine expression or protein release. Cytokine expression (left) was assessed by RT-qPCR normalized to $\beta$-actin. The data show fold changes in cytokine expression by neutrophils from I/NT and I/T mice as compared with naive mice and are representative of 3 independent experiments, with 8-10 mice per group. Protein release (right) was assessed by ELISA in supernatants of sorted neutrophils cultured at a density of 2 $\times 10^{5}$ cells/well for 24 hours. The data show BAFF and LT $\alpha$ release by neutrophils from I/ NT and $\mathrm{I} / \mathrm{T}$ and are representative of 3 independent experiments, with 8-10 mice per group. The dashed line represents the level of BAFF released by neutrophils sorted from naive mice. No LT $\alpha$ release was detected from neutrophils sorted from naive mice. (C) BAFF and LT $\alpha$ release by BM-isolated neutrophils. BAFF and $L T \alpha$ release was assessed by ELISA in supernatants of neutrophils isolated from BM of naive mice ( $>95 \%$ purity) and cultured for 24 hours in $667 \mathrm{mAb}$-coated 24-well plates at a density of $2 \times 10^{6}$ cells in $500 \mu \mathrm{l}$ medium. Experiments were done in the presence and in the absence of the proinflammatory cytokine IFN- $\gamma$ (100 ng/ ml). 667 mAb-noncoated plates were used as control. The data represent 4 independent experiments. Data are expressed as mean \pm SEM. Statistical significance was established using a parametric 1-way ANOVA test with a Bonferroni correction ( $\mathbf{A}$ and C) or a paired Student's $t$ test $(\mathbf{B})\left({ }^{*} P<0.05\right.$; ${ }^{* *} P<0.01 ;{ }^{* *} P<0.001$ ).

In summary, FrCasE infection activates neutrophils independently of immunotherapy. However, the activation state substantially differs between the two groups of animals, notably with a significantly stronger expression of LT $\alpha$ and BAFF in immunotherapy-treated mice. In addition, inflammatory conditions synergize with mAb-mediated activation of neutrophils leading to an enhanced release of BAFF and LT $\alpha$.

\section{Discussion}

We have previously shown that antiviral mAb-based therapies can induce life-long protective immunity. This finding potentially has important therapeutic implications, as evidence suggests that it may also apply to diverse severe human viral diseases $(10,14)$. A paramount task is now to identify the mechanisms at the origin of the mAb-induced vaccine-like effects and exploit them for more efficient mAb-based treatment of patients.

Here, we report that neutrophils are essential during the immunotherapy period for long-term survival of FrCasE-infected mice, not because they control viral propagation but because they are crucial for inducing a 
protective humoral response without an effect on the $\mathrm{CD} 8^{+} \mathrm{T}$ cell response. In contrast, we show that, upon $667 \mathrm{mAb}$ treatment, NK cells are crucial for the elimination of infected cells by $667 \mathrm{mAb}$-mediated ADCC activity and are necessary for long-term survival of infected/treated mice. Thus, our work indicates that both innate effector cells have distinct but complementary roles in the protection of infected mice by $\mathrm{mAb}$; NK cells have an early and predominant role in the control of viral spread, while neutrophils are essential for the emergence of a potent host antiviral humoral response. Our findings contrast with the current view of neutrophils - they are usually considered as simple frontline agents against invading pathogens - and highlight the hitherto unreported role of neutrophils as key cells in the modulation of adaptive antiviral immunity upon mAb treatment.

Our data indicate that survival of infected/treated mice depends on an efficient anti-FrCasE humoral response. Infected/treated mice show neutrophil-dependent (a) increased frequency of MZ B cells, (b) enhanced formation of GCs, (c) increased plasma cell generation, and (d) enhanced production of antiviral IgGs. Interestingly, the absence of neutrophils during the immunotherapy period in infected mice also leads to impaired development of secondary humoral responses upon viral challenge. Overall, our data suggest that neutrophils are essential for antiviral protection due to their B cell helper activity. Similarly, such a helper function has already been documented under homeostatic conditions (51), in autoimmunity disease-prone mice (52), during emergency granulopoiesis (53), and in bacterial infection (54). Importantly, our findings show that the acquisition of $\mathrm{B}$ cell helper functions in infected mice is dependent on immunotherapy, as no modulation of the anti-FrCasE humoral response was detected in infected/nontreated mice upon neutrophil depletion.

Though not excluding the role of other factors, our study suggests potential roles for BAFF and LT $\alpha$ in this B cell helper function. As concerns BAFF, it is interesting to note that its secretion by splenic neutrophils can contribute to the activation of splenic MZ B cells and the acceleration of plasma cell generation $(51,55)$. Moreover, BAFF administration to mice increases both the frequency of MZ B cells and antibody production $(56,57)$ and constitutes a signal for both MZ B cell survival and differentiation into plasmablasts $(47,58-61)$. Together with the fact that MZ B cells can favor the generation of plasma cells upon microbial infection (49), it is reasonable to speculate that BAFF induction in neutrophils of infected/treated mice may also favor the MZ B cell response and the subsequent generation of plasma cells. Interestingly, in agreement with our in vitro results showing enhanced BAFF release by neutrophils after a combination of IFN- $\gamma$ stimulation and Fc $\gamma \mathrm{R}$ cross-linking (Figure 7C), different proinflammatory stimuli, including ICs, have been shown to act as secretagogues and to synergize with IFN- $\gamma$ to enhance BAFF secretion by human neutrophils (62). Moreover, neutrophil depletion in autoimmunity-prone mice led to a reduction of autoantibodies titers that correlated with decreased serum levels of IFN- $\gamma$ and BAFF (52). Thus, it can reasonably be hypothesized that inflammatory conditions synergize with ICs formed after $667 \mathrm{mAb}$ treatment of FrCasE-infected mice to enhance BAFF release by neutrophils.

Although not formally shown, our data suggest that LT $\alpha$ release by neutrophils might play a role in the enhancement of the antiviral humoral response in infected/treated mice. LT $\alpha$ is involved in the formation of secondary lymphoid organs, is expressed by lymphocytes, and mediates a large variety of inflammatory and antiviral responses. It has also been reported to play a role in the development of GC formation and to be required for IgG responses (63-65). Here, we show that this cytokine is expressed and released by neutrophils upon $\mathrm{mAb}$ treatment of infected mice. Interestingly, although LT $\alpha$ is not known to be expressed by neutrophils, it has been proposed that nonlymphocytic murine splenic cells are able to produce it (66). We also report that Fc $\gamma \mathrm{R}$ cross-linking by immobilized $667 \mathrm{mAb}$ synergizes with IFN- $\gamma$ to enhance LT $\alpha$ release by neutrophils. This suggests that, similarly to BAFF, inflammatory conditions and $\mathrm{mAb}$ triggering might lead to increased LT $\alpha$ secretion by neutrophils. Further studies will be required to address whether BAFF and LT $\alpha$ combine their actions to stimulate the antiviral humoral response upon mAb immunotherapy. Finally, as IFN- $\gamma$ potentiates BAFF and LT $\alpha$ release, it will also be important to assess whether and how neutrophil-dependent IFN- $\gamma$ production by NK cells in infected/treated mice contributes to the stimulation of antibody responses, as this cytokine affects class-switching and long-term maintenance of neutralizing antibody titers in retrovirally infected mice (34) and humoral autoimmunity in humans (67).

Identifying neutrophils as key players in the induction of protective immunity by antiviral mAbs has important therapeutic implications for several reasons. First, if combination therapies are considered, it will be of utmost importance that the agent(s) coadministered with the passive immunotherapy do not alter neutrophil functions and counts to avoid inhibiting efficient antiviral humoral immune 
responses. Second, in pathological situations leading to neutropenia and/or impaired neutrophils functions, such as certain viral infections $(68,69)$ and/or drug-induced neutropenia $(70)$, it will be essential to restore them. This could, for example, be achieved through administration of granulocyte colony-stimulating factor (G-CSF), a cytokine already used in the clinic to treat neutropenic patients. Interestingly, this cytokine might also be used in combination with treatments aiming at enhancing the generation of functional neutrophils (70). Furthermore, beyond stimulating neutrophil activation and/or mobilization, G-CSF enhances neutrophil BAFF secretion capacity and, thereby, the ability of neutrophils to stimulate B cells (71). Similarly, reduction of viral load by antiretroviral therapies also permits partial restoration of the impaired functions of neutrophils observed in HIV-infected patients (68). Interestingly, neutrophils have been shown to mediate immunosuppression via the PD-L1/PD-1 pathway in HIV-infected patients (72). Antiviral passive immunotherapies might therefore benefit from combination with the administration of mAbs targeting this immune checkpoint. Finally, alternative approaches, such as engineering the Fc fragment of antiviral mAbs, merit consideration. Increasing their affinity for the Fc $\gamma$ Rs expressed by neutrophils might, at first, allow superior antibody-mediated phagocytosis, as recently reported in the case the Fc-modified VR01 anti-HIV mAb (73). This could then alter cell signaling and cytokine/chemokines production to ultimately lead to more effective adaptive immune responses. Thus, stimulating neutrophil activity, restoring their impaired functions, and/ or counteracting their immunosuppressive actions should improve the vaccine-like effects of antiviral mAb-based immunotherapies. This might also apply to cancer treatment as enhancement of antitumoral immune responses has also been observed in mAb-based anticancer immunotherapies. This is all the more important to take into consideration, as neutrophils have been shown to play a role in the therapeutic activity of anticancer mAb (74-78).

\section{Methods}

Mice. Mice were bred and maintained under conventional, pathogen-free facilities at the Institut de Génétique Moléculaire de Montpellier.

Viral stocks. FrCasE viral stocks were produced, assayed, and stored as described previously (27).

Viral infection, immunotherapy, and mouse follow-up. Eight-day-old 129/Sv/Ev mice were infected i.p. with $50 \mu \mathrm{l}$ of a virus suspension containing 50,000 focus-forming units (FFU) and treated, or not, with $30 \mu \mathrm{g} 667 \mathrm{mAb} 1$ hour p.i. and on days 2 and 5 p.i. by i.p. administration. Mice were examined at regular intervals for clinical signs of erythroleukemia (reduction of hematocrits). They were euthanized when their hematocrits reached $35 \%$ (experimental endpoint).

Flow cytometry. Spleen single-cell suspensions were obtained by mechanical dissociation of the organs in PBS. BM cell suspensions were obtained by dissection and PBS flushing of tibias and femurs. Cells were stained at $4^{\circ} \mathrm{C}$ using fluorochrome-conjugated antibodies against CD3e (145-2C11, BD Biosciences), CD4 (RM4-5, eBioscience), CD8 (Ly2, 53-6.7, eBioscience), CD11b (M1/70, eBioscience), CD11c (HL3, BD Biosciences), CD19 (1D3, eBioscience), CD21/35 (eBio8d9, eBioscience), CD23 (B3B4, eBioscience), CD27 (LG3A10, BioLegend), CD45.2 (104, BD Biosciences), CD45R/B220 (RA3-6B2, BD Biosciences), CD49b (DX5, BD Biosciences), CD62L (Ly22, MEL-14, BD Biosciences), CD117 (2B8, BD Biosciences), CD127 (SB/199, BD Biosciences), CD138 (281-2, BioLegend), F4/80 (BM8, BioLegend), Gr1 (RB6-8C5, BD Biosciences), IFN- $\gamma$ (XMG1.2, BD Biosciences), IgM (eB121-15F9, eBioscience), Ly6G (1A8, BD Biosciences), lineage (Ter119; Gr1; CD45R/B220; CD11c; F4/80; CD3e, BioLegend), and NKp46 (29A1.4, BD Biosciences). FrCasE-infected cells were assayed using an anti-Gag mAb (H34) (35) labeled with Alexa Fluor 647. Forward scatter area and forward scatter time-of-flight, as well as side scatter, were used to remove doublets from flow cytometry analyses. Cells were analyzed on FACSCanto II flow cytometer (BD Bioscience), and the data were analyzed using the FlowJo software (Tree Star).

ELISA of anti-FrCasE antibodies. Plasma anti-FrCasE Igs were assayed by ELISA as previously described (26, 27). Peroxidase-conjugated anti-mouse IgG or IgM rabbit antisera (Serotec) were used as secondary antibodies.

Virus challenge experiments. Infected/treated mice, depleted or not of neutrophils, were injected i.v. with $300 \mu \mathrm{l}$ of a $5 \times 10^{4} \mathrm{FFU} / \mathrm{ml}$ FrCasE suspension mixed to $2 \times 10^{6}$ FrCasE-infected splenocytes 3 months after the first infection. Blood samples were collected 1 week after challenge to assay endogenous anti-FrCasE IgG concentrations by ELISA.

In vivo depletion of $\mathrm{Ly}_{6} \mathrm{G}^{+}$and $N K$ cells. Neutrophils were depleted by administering a rat anti-Ly6G antibody (1A8; BioXcell), $(37,38)$ injected i.p. at different time points $(150 \mu \mathrm{g} /$ injection), or isotype control rat 
IgG (2A3; BioXcell). Neutrophil depletion was monitored by flow cytometry analysis of Gr $1^{\text {hi }}$ and CD $11 b^{+}$ cells. NK cells were depleted using the anti-asialo GM1 antibody (Wako Pure Chemical Industries Ltd.), injected i.p. at different time points ( $50 \mu 1$ /injection). This antibody has been used to study the in vivo functions of NK cells in mouse strains lacking the NK1.1 allotype, which is a feature of $129 \mathrm{~Sv} / \mathrm{Ev}$ mice (42).

In vivo cytolysis activity. Experiments were conducted as described previously $(27,79)$. Briefly, red blood cell-free splenocytes were recovered from 10-day-old FrCasE-infected, or noninfected, pups. Splenocytes from noninfected mice were labeled with the vital dye carboxyfluorescein succinimidyl ester (CFSE; Molecular Probes) at a concentration of $0.5 \mu \mathrm{M}\left(\mathrm{CFSE}^{\text {lo }}\right.$ cells). Splenocytes from infected mice were labeled with $5 \mu \mathrm{M}$ CFSE (CFSE $^{\text {hi }}$ cells) and preincubated, or not, with the $667 \mathrm{mAb}$ (the absence of $667 \mathrm{mAb}$ allowed quantification of spontaneous cell death). Both cell populations were mixed at a 1:1 ratio before retro-orbital administration to recipient mice. Cytolysis activity against infected splenocytes was calculated from the ratio of $\mathrm{CFSE}^{\text {lo }} / \mathrm{CFSE}^{\text {hi }}$ cells in spleen assayed by flow cytometry 5 hours later. To assess the contribution of NK cells and neutrophils to antibody-mediated cytolysis, $50 \mu 1$ of the anti-asialo GM1 or $200 \mu \mathrm{g}$ of the anti-Ly6G $1 \mathrm{~A} 8 \mathrm{mAb}$ were administered 1 day prior to the assay.

Flow cytometry assay of $C D 8^{+} T$ cells specific for FrCasE-infected cells. Splenocytes were labeled with both an APC-conjugated anti-CD8 ${ }^{+} \mathrm{T}$ cell antibody and a PE-conjugated MHC class I H-2 $\mathrm{D}^{\mathrm{b}}$ tetramer (Beckman Coulter) displaying the immunodominant Friend virus GagL epitope $(27,80)$ ( $\mathrm{D}^{\mathrm{b}}$-GagL tetramers) as previously described (27).

Assay of IFN- $\gamma$ production. $10^{6}$ splenocytes were incubated at $37^{\circ} \mathrm{C}$ for 5 hours in 12 -well plates in $500 \mu 1$ RPMI culture medium containing phorbol 12 -myristate 13 -acetate $(50 \mathrm{ng} / \mathrm{ml})$ and ionomycin $(500 \mathrm{ng} / \mathrm{ml})$ in the presence of brefeldin A $(10 \mu \mathrm{g} / \mathrm{ml}$; MilliporeSigma). IFN- $\gamma$ production was assayed by flow cytometry using the intracellular Cytofix/Cytoperm Fixation/Permeabilization staining kit (Becton Dickinson).

Histological analyses. Spleens from infected/nontreated and infected/treated mice (depleted or not of neutrophils) were recovered at day 14 p.i. and prepared as previously described (81). Briefly, spleens were initially fixed at $4^{\circ} \mathrm{C}$ overnight with a paraformaldehyde-lysine-periodate (PLP) solution and then embedded in 4\% low-temperature-gelling agarose (type VII-1; MilliporeSigma) prepared in PBS. 300- $\mu$ m slices were cut with a vibratome (VT 1000S; Leica) in a bath of ice-cold PBS. Sections of tissues were submerged in PBS and transferred to 0.4- $\mu \mathrm{m}$ organotypic culture inserts (Millicell; Millipore) for staining with an anti-B220 (RA3-6B2, Becton Dickinson) and an anti-CD169 antibody (MOMA-1, Bio-Rad) at $37^{\circ} \mathrm{C}$ for 20 minutes. The images were captured using a Leica SP8-UV confocal scanning microscope.

$R T-q P C R$ quantification of gene expression. Single-cell suspensions of splenocytes were prepared from naive, infected/nontreated, and infected/treated mice 8 days p.i. and immunotherapy. Neutrophils (CD11b ${ }^{+}$Ly6G ${ }^{\text {h }}$ expression) were sorted (>98\% pure) using a BD Biosciences FACSAria device. RNA was extracted from 1 $\times 10^{6}$ to $2 \times 10^{6}$ sorted neutrophils using the RNeasy micro kit (Qiagen). RNA quality and integrity were verified using the Agilent 2100 bioanalyzer. cDNAs were synthesized using the RT2 First-Strand Kit (Qiagen). All quantitative PCRs were performed following protocols optimized for the RT2 quantitative Profiler PCR array using SYBR Green mix (Qiagen) and LightCycler 480 II machine (Roche). All data were normalized to $\beta$-actin. Results were expressed as fold increases with respect to naive cells using the $\Delta \Delta \mathrm{Ct}$ method.

$B A F F$ and LT $\alpha$ protein release quantification. Soluble BAFF and LT $\alpha$ from cell-free supernatants of cultured neutrophils were assayed using BAFF (R\&D Systems) and LT $\alpha$ ELISA (NeoBiotech), respectively. Supernatants were collected from sorted splenic neutrophils (from naive, infected/nontreated, and infected/treated mice at 8 days p.i. and immunotherapy) cultured in 96-well plates at a density of $2 \times 10^{5}$ cells/ well for 24 hours. Alternatively, neutrophils were isolated from naive mice BM using a magnetic-based cell sorting (MACS) neutrophil isolation kit (>95\% purity; Miltenyi Biotec) and cultured for 24 hours in 667 mAb-coated 24-well plates at a density of $2 \times 10^{6}$ cells in $500 \mu 1$ medium, in the presence or in the absence of IFN- $\gamma(100 \mathrm{ng} / \mathrm{ml}) .667 \mathrm{mAb}$-noncoated plates were used as controls. G-CSF (R\&D Systems) was added at a concentration of $10 \mathrm{ng} / \mathrm{ml}$ to neutrophil cultures to maintain cell viability.

Statistics. Statistical analyses were performed using GraphPad Prism 5 (GraphPad Software). Data were expressed as mean \pm SEM, and statistical significance was established using a parametric 1-way ANOVA test with a Bonferroni correction for multiple comparisons or unpaired Student's 2-tailed $t$ tests when two groups were compared. $P$ values lower than 0.05 were considered as statistically significant.

Study approval. All experimental procedures were performed in accordance with the French national animal care guidelines (CEEA-LR-12146 approval, Ethics committee of the Languedoc-Roussillon Region, Montpellier). 


\section{Author contributions}

M. Pelegrin, MNG, and M. Piechaczyk defined the research program. MNG, JL, and M. Pelegrin performed the experiments and carried out the data analyses, with a contribution by M. Piechaczyk. M. Pelegrin, MNG, and M. Piechaczyk wrote the manuscript.

\section{Acknowledgments}

This work was supported by grants from the Ligue Nationale Contre le Cancer (EL2015.LNCC/MaP), the Fondation ARC (SFI20121205665, contract 089287), Sidaction (BI25-1-02278 and A014-2-AEQ-08-01), and the Fondation pour la Recherche Médicale (SPF20120523949). MNG, JL, M. Piechaczyk, and M. Pelegrin are members of the "MabImprove Labex," a public grant overseen by the French National Research Agency as part of the "Investments for the future" program (reference ANR-10-LABX-53-01) that also supported this work. We thank the imaging facility MRI, which is part of the UMS BioCampus Montpellier and a member of the national infrastructure France-BioImaging, supported by the French National Research Agency (ANR-10-INBS-04, "Investments for the future"). We are grateful to the animal facility of the Institut de Génétique Moléculaire de Montpellier, which is part of the "Réseau des Animaleries Montpelliéraines" RAMIBiSA Facility for animal experiments, and to the "Réseau d'Histologie Experimentale de Montpellier" RHEM facility for expert assistance with histology. We are grateful to E. Donnadieu (Institut Cochin, Paris) for expert assistance with histology, to M. Boyer and S. Gailhac from MRI for support in cytometry experiments, to Thierry Gostan (SERENAD Complex Biological Data Analysis Service) for support in statistical analyses, to Helen Phillips Bevis (Traductions Stratégiques) for English editing services, and to V. Dardalhon, M. Hahne, and B. Hipskind for critical reading of the manuscript.

Address correspondence to: Mireia Pelegrin, Institute of Molecular Genetics of Montpellier, 1919, route de Mende, 34293 Montpellier Cedex 5, France. Phone: 33.4.34.35.96.68; Email: mireia.pelegrin@igmm.cnrs.fr.

1. Salazar G, Zhang N, Fu TM, An Z. Antibody therapies for the prevention and treatment of viral infections. NPJ Vaccines. 2017;2:19.

2. Both L, Banyard AC, van Dolleweerd C, Wright E, Ma JK, Fooks AR. Monoclonal antibodies for prophylactic and therapeutic use against viral infections. Vaccine. 2013;31(12):1553-1559.

3. Caskey M, et al. Antibody 10-1074 suppresses viremia in HIV-1-infected individuals. Nat Med. 2017;23(2):185-191.

4. Corti D, Lanzavecchia A. Broadly neutralizing antiviral antibodies. Annu Rev Immunol. 2013;31:705-742.

5. Corti D, et al. Prophylactic and postexposure efficacy of a potent human monoclonal antibody against MERS coronavirus. Proc Natl Acad Sci USA. 2015;112(33):10473-10478.

6. Corti D, et al. Protective monotherapy against lethal Ebola virus infection by a potently neutralizing antibody. Science. 2016;351(6279):1339-1342.

7. De Benedictis P, et al. Development of broad-spectrum human monoclonal antibodies for rabies post-exposure prophylaxis EMBO Mol Med. 2016;8(4):407-421.

8. Fibriansah $\mathrm{G}$, et al. A potent anti-dengue human antibody preferentially recognizes the conformation of $\mathrm{E}$ protein monomers assembled on the virus surface. EMBO Mol Med. 2014;6(3):358-371.

9. Lynch RM, et al. Virologic effects of broadly neutralizing antibody VRC01 administration during chronic HIV-1 infection. Sci Transl Med. 2015;7(319):319ra206.

10. Pelegrin M, Naranjo-Gomez M, Piechaczyk M. Antiviral monoclonal antibodies: Can they be more than simple neutralizing agents? Trends Microbiol. 2015;23(10):653-665.

11. Bangaru S, et al. Recognition of influenza H3N2 variant virus by human neutralizing antibodies. JCI Insight. 2016;1(10):e86673

12. Bailey JR, et al. Broadly neutralizing antibodies with few somatic mutations and hepatitis C virus clearance. JCI Insight. 2017;2(9):92872.

13. Kam YW, et al. Cross-reactive dengue human monoclonal antibody prevents severe pathologies and death from Zika virus infections. JCI Insight. 2017;2(8):92428.

14. Schoofs T, et al. HIV-1 therapy with monoclonal antibody 3BNC117 elicits host immune responses against HIV-1. Science. 2016;352(6288):997-1001

15. Lambour J, Naranjo-Gomez M, Piechaczyk M, Pelegrin M. Converting monoclonal antibody-based immunotherapies from passive to active: bringing immune complexes into play. Emerg Microbes Infect. 2016;5(8):e92.

16. Wen YM, Mu L, Shi Y. Immunoregulatory functions of immune complexes in vaccine and therapy. EMBO Mol Med. 2016;8(10):1120-1133.

17. Abès R, Gélizé E, Fridman WH, Teillaud JL. Long-lasting antitumor protection by anti-CD20 antibody through cellular immune response. Blood. 2010;116(6):926-934.

18. Deligne C, Metidji A, Fridman WH, Teillaud JL. Anti-CD20 therapy induces a memory Th1 response through the IFN- $\gamma /$ IL-12 axis and prevents protumor regulatory T-cell expansion in mice. Leukemia. 2015;29(4):947-957.

19. DiLillo DJ, Ravetch JV. Differential Fc-receptor engagement drives an anti-tumor vaccinal effect. Cell. 2015;161(5):1035-1045.

20. Hilchey SP, et al. Rituximab immunotherapy results in the induction of a lymphoma idiotype-specific T-cell response in patients 
with follicular lymphoma: support for a "vaccinal effect" of rituximab. Blood. 2009;113(16):3809-3812.

21. Srivastava RM, et al. Cetuximab-activated natural killer and dendritic cells collaborate to trigger tumor antigen-specific T-cell immunity in head and neck cancer patients. Clin Cancer Res. 2013;19(7):1858-1872.

22. Taylor C, et al. Augmented HER-2 specific immunity during treatment with trastuzumab and chemotherapy. Clin Cancer Res. 2007;13(17):5133-5143.

23. Trivedi S, et al. Anti-EGFR targeted monoclonal antibody isotype influences antitumor cellular immunity in head and neck cancer patients. Clin Cancer Res. 2016;22(21):5229-5237.

24. Gros L, Dreja H, Fiser AL, Plays M, Pelegrin M, Piechaczyk M. Induction of long-term protective antiviral endogenous immune response by short neutralizing monoclonal antibody treatment. J Virol. 2005;79(10):6272-6280.

25. Gros L, Pelegrin M, Plays M, Piechaczyk M. Efficient mother-to-child transfer of antiretroviral immunity in the context of preclinical monoclonal antibody-based immunotherapy. J Virol. 2006;80(20):10191-10200.

26. Gros L, et al. Endogenous cytotoxic T-cell response contributes to the long-term antiretroviral protection induced by a short period of antibody-based immunotherapy of neonatally infected mice. $J$ Virol. 2008;82(3):1339-1349.

27. Michaud HA, et al. A crucial role for infected-cell/antibody immune complexes in the enhancement of endogenous antiviral immunity by short passive immunotherapy. PLoS Pathog. 2010;6(6):e1000948.

28. Nasser R, Pelegrin M, Michaud HA, Plays M, Piechaczyk M, Gros L. Long-lasting protective antiviral immunity induced by passive immunotherapies requires both neutralizing and effector functions of the administered monoclonal antibody. $J$ Virol. 2010;84(19):10169-10181.

29. Nasser R, Pelegrin M, Plays M, Gros L, Piechaczyk M. Control of regulatory T cells is necessary for vaccine-like effects of antiviral immunotherapy by monoclonal antibodies. Blood. 2013;121(7):1102-1111.

30. Mócsai A. Diverse novel functions of neutrophils in immunity, inflammation, and beyond. J Exp Med. 2013;210(7):1283-1299

31. Scapini P, Cassatella MA. Social networking of human neutrophils within the immune system. Blood. 2014;124(5):710-719.

32. Galani IE, Andreakos E. Neutrophils in viral infections: Current concepts and caveats. J Leukoc Biol. 2015;98(4):557-564.

33. Saitoh T, et al. Neutrophil extracellular traps mediate a host defense response to human immunodeficiency virus-1. Cell Host Microbe. 2012;12(1):109-116.

34. Stromnes IM, et al. Temporal effects of gamma interferon deficiency on the course of Friend retrovirus infection in mice. J Virol. 2002;76(5):2225-2232.

35. Chesebro B, et al. Characterization of mouse monoclonal antibodies specific for Friend murine leukemia virus-induced erythroleukemia cells: friend-specific and FMR-specific antigens. Virology. 1981;112(1):131-144.

36. Dittmer U, Race B, Peterson KE, Stromnes IM, Messer RJ, Hasenkrug KJ. Essential roles for CD8+ T cells and gamma interferon in protection of mice against retrovirus-induced immunosuppression. J Virol. 2002;76(1):450-454.

37. Carr KD, Sieve AN, Indramohan M, Break TJ, Lee S, Berg RE. Specific depletion reveals a novel role for neutrophil-mediated protection in the liver during Listeria monocytogenes infection. Eur J Immunol. 2011;41(9):2666-2676.

38. Daley JM, Thomay AA, Connolly MD, Reichner JS, Albina JE. Use of Ly6G-specific monoclonal antibody to deplete neutrophils in mice. J Leukoc Biol. 2008;83(1):64-70.

39. Bruel T, et al. Elimination of HIV-1-infected cells by broadly neutralizing antibodies. Nat Commun. 2016;7:10844.

40. Smalls-Mantey A, Connors M, Sattentau QJ. Comparative efficiency of HIV-1-infected T cell killing by NK cells, monocytes and neutrophils. PLoS ONE. 2013;8(9):e74858.

41. Bodhankar S, Woolard MD, Sun X, Simecka JW. NK cells interfere with the generation of resistance against mycoplasma respiratory infection following nasal-pulmonary immunization. J Immunol. 2009;183(4):2622-2631.

42. Carlyle JR, et al. Molecular and genetic basis for strain-dependent NK1.1 alloreactivity of mouse NK cells. J Immunol. 2006;176(12):7511-7524.

43. Kasai M, Iwamori M, Nagai Y, Okumura K, Tada T. A glycolipid on the surface of mouse natural killer cells. Eur J Immunol. 1980;10(3):175-180

44. Ong S, et al. Natural killer cells limit cardiac inflammation and fibrosis by halting eosinophil infiltration. Am J Pathol. 2015;185(3):847-861.

45. Jaeger BN, et al. Neutrophil depletion impairs natural killer cell maturation, function, and homeostasis. JExp Med. 2012;209(3):565-580.

46. Cortez VS, Robinette ML, Colonna M. Innate lymphoid cells: new insights into function and development. Curr Opin Immunol. 2015;32:71-77.

47. Magri G, et al. Innate lymphoid cells integrate stromal and immunological signals to enhance antibody production by splenic marginal zone B cells. Nat Immunol. 2014;15(4):354-364.

48. Cerutti A, Cols M, Puga I. Marginal zone B cells: virtues of innate-like antibody-producing lymphocytes. Nat Rev Immunol. 2013;13(2):118-132.

49. Song H, Cerny J. Functional heterogeneity of marginal zone B cells revealed by their ability to generate both early antibody-forming cells and germinal centers with hypermutation and memory in response to a T-dependent antigen. J Exp Med. 2003;198(12):1923-1935.

50. Zouali M, Richard Y. Marginal zone B-cells, a gatekeeper of innate immunity. Front Immunol. 2011;2:63.

51. Puga I, et al. B cell-helper neutrophils stimulate the diversification and production of immunoglobulin in the marginal zone of the spleen. Nat Immunol. 2011;13(2):170-180.

52. Coquery CM, et al. Neutrophils contribute to excess serum BAFF levels and promote CD4+ T cell and B cell responses in lupus-prone mice. PLoS One. 2014;9(7):e102284.

53. Parsa R, et al. BAFF-secreting neutrophils drive plasma cell responses during emergency granulopoiesis. JExp Med. 2016;213(8):1537-1553.

54. Chorny A, et al. The soluble pattern recognition receptor PTX3 links humoral innate and adaptive immune responses by helping marginal zone B cells. J Exp Med. 2016;213(10):2167-2185.

55. Cerutti A, Puga I, Magri G. The B cell helper side of neutrophils. J Leukoc Biol. 2013;94(4):677-682.

56. Dosenovic P, et al. BLyS-mediated modulation of naive B cell subsets impacts HIV Env-induced antibody responses. J Immunol. 
2012;188(12):6018-6026.

57. Enoksson SL, et al. The inflammatory cytokine IL-18 induces self-reactive innate antibody responses regulated by natural killer T cells. Proc Natl Acad Sci USA. 2011;108(51):E1399-E1407.

58. Lopes-Carvalho T, Foote J, Kearney JF. Marginal zone B cells in lymphocyte activation and regulation. Curr Opin Immunol. 2005;17(3):244-250.

59. McCulloch L, Smith CJ, McColl BW. Adrenergic-mediated loss of splenic marginal zone B cells contributes to infection susceptibility after stroke. Nat Commun. 2017;8:15051.

60. Srivastava B, Quinn WJ, Hazard K, Erikson J, Allman D. Characterization of marginal zone B cell precursors. J Exp Med. 2005;202(9):1225-1234.

61. Schweighoffer E, et al. The BAFF receptor transduces survival signals by co-opting the B cell receptor signaling pathway. Immunity. 2013;38(3):475-488.

62. Scapini P, et al. Proinflammatory mediators elicit secretion of the intracellular B-lymphocyte stimulator pool (BLyS) that is stored in activated neutrophils: implications for inflammatory diseases. Blood. 2005;105(2):830-837.

63. Matsumoto M, Fu YX, Molina H, Chaplin DD. Lymphotoxin-alpha-deficient and TNF receptor-I-deficient mice define developmental and functional characteristics of germinal centers. Immunol Rev. 1997;156:137-144.

64. Banks TA, et al. Lymphotoxin-alpha-deficient mice. Effects on secondary lymphoid organ development and humoral immune responsiveness. J Immunol. 1995;155(4):1685-1693.

65. Fu YX, Molina H, Matsumoto M, Huang G, Min J, Chaplin DD. Lymphotoxin-alpha (LTalpha) supports development of splenic follicular structure that is required for IgG responses. J Exp Med. 1997;185(12):2111-2120.

66. Fu YX, Huang G, Wang Y, Chaplin DD. Lymphotoxin-alpha-dependent spleen microenvironment supports the generation of memory B cells and is required for their subsequent antigen-induced activation. J Immunol. 2000;164(5):2508-2514.

67. Jackson SW, et al. B cell IFN- $\gamma$ receptor signaling promotes autoimmune germinal centers via cell-intrinsic induction of BCL-6. J Exp Med. 2016;213(5):733-750.

68. Casulli S, Elbim C. Interactions between human immunodeficiency virus type 1 and polymorphonuclear neutrophils. J Innate Immun. 2014;6(1):13-20.

69. Shi X, et al. Neutropenia during HIV infection: adverse consequences and remedies. Int Rev Immunol. 2014;33(6):511-536.

70. Li L, et al. Am80-GCSF synergizes myeloid expansion and differentiation to generate functional neutrophils that reduce neutropenia-associated infection and mortality. EMBO Mol Med. 2016;8(11):1340-1359.

71. Scapini P, et al. G-CSF-stimulated neutrophils are a prominent source of functional BLyS. J Exp Med. 2003;197(3):297-302.

72. Bowers NL, Helton ES, Huijbregts RP, Goepfert PA, Heath SL, Hel Z. Immune suppression by neutrophils in HIV-1 infection: role of PD-L1/PD-1 pathway. PLoS Pathog. 2014;10(3):e1003993.

73. Sips M, et al. Fc receptor-mediated phagocytosis in tissues as a potent mechanism for preventive and therapeutic HIV vaccine strategies. Mucosal Immunol. 2016;9(6):1584-1595.

74. Albanesi M, et al. Neutrophils mediate antibody-induced antitumor effects in mice. Blood. 2013;122(18):3160-3164

75. Cittera E, et al. The CCL3 family of chemokines and innate immunity cooperate in vivo in the eradication of an established lymphoma xenograft by rituximab. J Immunol. 2007;178(10):6616-6623.

76. Golay J, et al. Glycoengineered CD20 antibody obinutuzumab activates neutrophils and mediates phagocytosis through CD16B more efficiently than rituximab. Blood. 2013;122(20):3482-3491.

77. Hernandez-Ilizaliturri FJ, et al. Neutrophils contribute to the biological antitumor activity of rituximab in a non-Hodgkin's lymphoma severe combined immunodeficiency mouse model. Clin Cancer Res. 2003;9(16 Pt 1):5866-5873.

78. Valgardsdottir R, Cattaneo I, Klein C, Introna M, Figliuzzi M, Golay J. Human neutrophils mediate trogocytosis rather than phagocytosis of CLL B cells opsonized with anti-CD20 antibodies. Blood. 2017;129(19):2636-2644.

79. Guyre CA, Gomes D, Smith KA, Kaplan JM, Perricone MA. Development of an in vivo antibody-mediated killing (IVAK) model, a flow cytometric method to rapidly evaluate therapeutic antibodies. J Immunol Methods. 2008;333(1-2):51-60.

80. Chen W, Qin H, Chesebro B, Cheever MA. Identification of a gag-encoded cytotoxic T-lymphocyte epitope from FBL-3 leukemia shared by Friend, Moloney, and Rauscher murine leukemia virus-induced tumors. J Virol. 1996;70(11):7773-7782.

81. Peranzoni E, et al. Ex vivo imaging of resident CD8 T lymphocytes in human lung tumor slices using confocal microscopy. $J$ Vis Exp. 2017;(130) 